\title{
Geometry of Superconformal Manifolds
}

\author{
A. A. Rosly, A. S. Schwarz, and A. A. Voronov \\ Institute of Theoretical and Experimental Physics, SU-117259 Moscow, USSR \\ To the memory of our friend and colleague Vadim Knizhnik
}

\begin{abstract}
The main facts about complex curves are generalized to superconformal manifolds. The results thus obtained are relevant to the fermion string theory and, in particular, they are useful for computation of determinants of super laplacians which enter the string partition function.
\end{abstract}

\section{Introduction}

The computation of fermion string amplitudes in the Polyakov formalism [1] reduces to integration over a finite-dimensional superspace, the space of classes of superconformal manifolds [2]. Superconformal manifolds were introduced in [2], and after that in an independent paper [3] they were introduced under the name "super Riemann manifolds." (Note that we reserve the name "super Riemann manifold" for a different usage, following the terminology of [4]. Reference [4] contains also a detailed account of part of the results of [2] and of later work $[8,9]$.) In $[2]$ the space of classes of superconformal manifolds was described. It was shown also that in computing the fermion string partition function a measure arose on this superconformal moduli space which was written in terms of determinants of certain operators analogous to the Laplacian. In [8] these determinants were expressed via a super analog of Selberg's zeta-function. (See [9] for details. The corresponding expression for the bosonic string was obtained first in [2].) Our purpose is now to study analytical properties of string measure on the moduli superspace and to find formulae for determinants of superlaplacians and for this measure. It is worthwhile to note that the analytical properties of the fermion string measure are essential in establishing the connection of this theory with the superstring theory of Green and Schwarz (see [12]) and with the heterotic string theory. (Note also that a supermoduli space relevant to the heterotic string was considered in [5].)

The background for our considerations will be the geometry of superconformal manifolds which is the subject of the present paper. Applications to string theory will be given in a following paper [13], the results of which are recapitulated below. 
A superconformal manifold is a (1|1)-dimensional complex supermanifold composed of superdomains glued together by superconformal transformations (that is to say, by transformations preserving the operator $D=\partial / \partial \zeta+\zeta \partial / \partial z$ up to a factor). We consider holomorphic line bundles over a superconformal manifold $\mathscr{N}$. Among such bundles an important one is the bundle $\omega$, as well as its tensor powers $\omega^{k}$, such that the field $D \varphi$ is a section of $\omega$ if $\varphi$ is a scalar function. Let us denote by $\mathscr{A}(L)$ the space of holomorphic sections of a bundle $L$. If the superconformal manifold $\mathscr{N}$ is simply connected, then every $\psi \in \mathscr{A}(\omega)$ can be represented as $\psi=D \varphi$ for a holomorphic function $\varphi$. For a non-simply connected $\mathscr{N}$ such a representation is possible only in terms of universal covering. (In other words, one has to consider the multivalued function $\varphi$.) The differences of the values of $\varphi$ between various pairs of points in the universal covering, such that both points in a pair correspond to the same point of $\mathscr{N}$, are called the periods of $\psi \in \mathscr{A}(\omega)$. If $\psi$ is a meromorphic section of the bundle $\omega$, then over a portion of $\mathscr{N}$ it can be considered as a holomorphic section, and so one is able to consider the periods of a meromorphic section. The periods that correspond to the changes of $\varphi$ when turning around the poles of $\psi$ are called (up to a factor of $2 \pi i$ ) the residues of $\psi$. (Here $\psi=D \varphi$ as before.) One can prove some statements about the periods and residues analogous to the case of algebraic curves, in particular, Riemann relations and Weil reciprocity law (see Sect. 2). It turns out that these proofs are easier to derive not in terms of the above definition of periods, but using anothwr one based on a relation (Sect. 1) between the fields considered above and differential forms on a superconformal manifold. Taking the quotient of $\mathscr{A}(\omega)^{*}$ by the period lattice one constructs the Jacobi manifold $J(\mathscr{N})$ for a compact superconformal manifold $\mathscr{N}$. By a divisor on $\mathscr{N}$ a formal linear combination, $\sum n_{i} P_{i}$, of the points $P_{i} \in \mathscr{N}$ with integer coefficients $n_{i}$ is meant. The sum of coefficients $n_{i}$ is called the degree of a divisor. A point $P \in \mathscr{N}$ is called the principal zero of a meromorphic section $s$ if $s(P)=0$ and $D s(P)=0$. A principal zero of $s^{-1}$ is called a principal pole of $s$. If a section $s$ possesses only simple zeros $Q_{i}$ and poles $P_{i}$, then the divisor of this section is $\sum Q_{i}-\sum P_{i}$. The set of divisors is divided in classes of linear equivalence. (The divisor of a meromorphic function is referred to as a divisor linearly equivalent to zero.) One can prove that topologically trivial holomorphic line bundles over $\mathscr{N}$ can be described by means of $J(\mathscr{N})$, or by means of the group made up of classes of divisors which have zero degree (Sect. 4). These statements can be thought of as a superanalog of the Abel-Jacobi theorem. In Sect. 5 the cohomologies with coefficients in a line bundle are computed and an analog of the Riemann-Roch theorem is proved. The paper ends with an Appendix where the basic definitions used are made more accurate. In a following paper [13] the results of the present work are applied to a study of string measure on superconformal moduli space. That paper begins with a consideration of holomorphic hermitian bundles over a superconformal manifold $\mathscr{N}$. A super Riemann metric on $\mathscr{N}$, according to one of possible definitions, is a hermitian structure on the bundle $\omega$. If $L$ is an hermitian bundle over a super Riemann manifold, then one is able to define an inner product in the space, $\Gamma(L)$, of sections of $L$ and by means of this inner product to construct the Laplacian $\square_{L}=\bar{D}^{+} \bar{D}$, where $\bar{D}=\partial / \partial \bar{\zeta}+\bar{\zeta} \partial / \partial \bar{z}$ is considered as an operator acting from $\Gamma(L)$ into $\Gamma(L \otimes \bar{\omega})$. The following expression for $\operatorname{det} \square_{L}$ can be 
considered as our main result [13]:

$$
\operatorname{det} \square_{L}=\frac{\operatorname{det} N_{L} \operatorname{det} N_{\mathcal{O}}^{\prime}}{\operatorname{det} N_{L}^{\prime} \cdot \int_{\mathscr{N}} d v}\left|R_{\mathcal{O}, L}(s, \sigma)\right|^{-2}\{s, \sigma\}^{2} \operatorname{det} \square .
$$

Here $d v$ is the volume element corresponding to the super Riemann metric ${ }^{1}, L$ is an arbitrary hermitian line bundle over a normal super-Riemann manifold $\mathscr{N}$ (see Sect. 2), $\mathcal{O}$ is a trivial line bundle with the direct product metric. We assume that an even holomorphic section $s$ of $L$ is given such that $s_{\text {red }}$ has only simple zeros. Let us denote the principal simple zeros of $s$ by $Q_{1}, \ldots, Q_{N}$. We suppose that $\mathscr{A}(L)$ and $\mathscr{A}\left(L^{*} \otimes \omega\right)$ can be considered as superspaces. (This terminology is explained in the Appendix.) Here $L^{*}$ is the dual bundle. That is to say, one can choose bases $\left\{\alpha_{1}, \ldots, \alpha_{m}\right\}$ in $\mathscr{A}(L)$ and $\left\{\beta_{1}, \ldots, \beta_{n}\right\}$ in $\mathscr{A}\left(L^{*} \otimes \omega\right)$. We shall prove that $m \geqq 1$ and $n \leqq g$. The frame $\left\{\alpha_{i}\right\}$ is picked so that $s=\alpha_{m}$, while a frame $\left\{\omega_{1}, \ldots, \omega_{g}\right\}$ in $\mathscr{A}(\omega)$ is required to obey: $\omega_{g-n+1}=s \beta_{1}, \ldots, \omega_{g}=s \beta_{n}$. In Eq. (1) $N_{L}, N_{L}^{\prime}$, and $N_{\mathcal{O}}^{\prime}$ denote the matrices of inner products of elements of frames chosen in $\mathscr{A}(L), \mathscr{A}\left(L^{*} \otimes \omega\right)$, and $\mathscr{A}(\omega)$ respectively. Namely, $\left(N_{L}\right)_{i j}=\left\langle\alpha_{i}, \alpha_{j}\right\rangle=\int\left(\alpha_{i}, \alpha_{j}\right) d v, \quad\left(N_{L}^{\prime}\right)_{i j}=\left\langle\beta_{i}, \beta_{j}\right\rangle$ $=\int\left(\beta_{i}, \beta_{j}\right) d v,\left(N_{\mathcal{O}}^{\prime}\right)_{i j}=\left\langle\omega_{i}, \omega_{j}\right\rangle=\int\left(\omega_{i}, \omega_{j}\right) d v$, where $($,$) is the hermitian product in$ the fibres of bundles $L, L^{*} \otimes \omega$, and $\omega$ respectively. $R_{\mathscr{O}, L}(s, \sigma)$ in Eq. (1) denotes $\operatorname{det}\left(\begin{array}{c}A \\ { }^{t} B^{-1}\end{array}\right)$, where

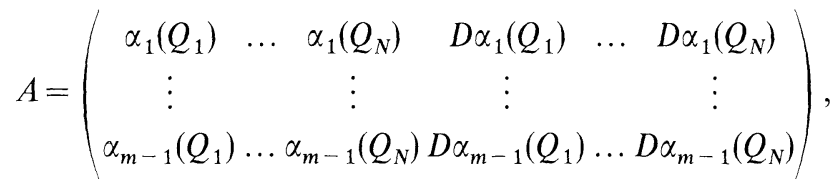

$$
\begin{aligned}
& B=\left(\begin{array}{cccccc}
\operatorname{res} \omega_{1} / s & \ldots & \operatorname{res} \omega_{1} / s & \operatorname{res} \zeta^{(1)} \omega_{1} / s & \ldots & \operatorname{res} \zeta^{(N)} \omega_{1} / s \\
Q_{1} & & Q_{N} & Q_{1} & & Q_{N} \\
\vdots & & \vdots & \vdots & & \vdots \\
\operatorname{res} \omega_{g-n} / s & \ldots & \operatorname{res} \omega_{g-n} / s & \operatorname{res} \zeta^{(1)} \omega_{g-n} / s & \ldots & \operatorname{res} \zeta^{(N)} \omega_{g-n} / s \\
Q_{1} & & Q_{N} & Q_{1} & & Q_{N}
\end{array}\right)
\end{aligned}
$$

Here it is supposed that in a neighbourhood of each point $Q_{i}$ one has chosen a superconformal coordinate system $\left(z^{(i)}, \zeta^{(i)}\right)$ with the origin in $Q_{i}$ and a trivialization of $L$. Then res $\gamma=b_{-1}$ if $\gamma=\sum_{Q_{i}}^{+\infty}\left(z^{(i)}\right)^{k}\left[a_{k}+\zeta^{(i)} b_{k}\right]$. Finally, $\sigma$ in Eq. (1) denotes an even meromorphic section of $\omega$, such that $\sigma\left(Q_{i}\right)=1$ for every $i$. Then $\{s, \sigma\}$ is defined by

$$
\{s, \sigma\}=\exp \left(\int R \cdot \log \|s\|-\sum \log \left\|\sigma\left(Q_{i}\right)\right\|\right),
$$

where $R$ is the curvature of the super Riemann manifold, that is the curvature of the hermitian bundle $\omega$. (For an arbitrary hermitian bundle, the curvature $F$ is defined by the expression $F=D \bar{D} \log \|s\|$, where $s$ is an arbitrary local holomorphic section.)

\footnotetext{
${ }^{1}$ One has to take such a super Riemann metric on $\mathscr{N}$ that $\int d v$ is an invertible element of the Grassmann algebra. For example, this can be the super-Poincare metric [9] with $d v=\left(\operatorname{Im} z+\frac{i}{2} \zeta \bar{\zeta}\right)^{-1} d z d \bar{z} d \zeta d \bar{\zeta}$ or the metric given in [12]
} 
The formula (1) leads immediately to the following important relation:

$$
\frac{\operatorname{det} \square_{L \otimes M}}{\operatorname{det} \square_{\mathcal{O}}}=\frac{\operatorname{det} \square_{L}}{\operatorname{det} \square_{\mathcal{O}}} \cdot \frac{\operatorname{det} \square_{M}}{\operatorname{det} \square_{\mathcal{O}}} \cdot B_{L, M}^{-1},
$$

where $L, M$ are arbitrary hermitian bundles. If $M$ has an even holomorphic section $s$ with simple principal zeros and if $\mathscr{A}(L), \mathscr{A}\left(L^{*} \otimes \omega\right), \mathscr{A}(M), \mathscr{A}\left(M^{*} \otimes \omega\right), \mathscr{A}(L \otimes M)$, $\mathscr{A}\left(L^{*} \otimes M^{*} \otimes \omega\right)$ can be considered as superspaces, then

$$
B_{L, M}^{-1}=\frac{\operatorname{det} N_{L \otimes M} \operatorname{det} N_{L}^{\prime} \operatorname{det} N_{M}^{\prime} \cdot\left(\int d v\right) \cdot\left|R_{\mathcal{O}, L}(s, \sigma)\right|^{2}}{\operatorname{det} N_{L \otimes M}^{\prime} \operatorname{det} N_{L} \operatorname{det} N_{M} \operatorname{det} N_{\mathscr{O}}^{\prime}\left|R_{M, L \otimes M}(s, \sigma)\right|^{2}} .
$$

In a general case, the expression for $B_{L, M}^{-1}$ becomes more complicated. Equation (6) yields readily an expression for string measure. In fact,

$$
\frac{\operatorname{det} \square_{\omega^{3}}}{\operatorname{det} \square_{\mathcal{O}}}=\frac{\operatorname{det} \square_{\omega^{2}}}{\operatorname{det} \square_{\mathcal{O}}} \cdot \frac{\operatorname{det} \square_{\omega}}{\operatorname{det} \square_{\mathcal{O}}} \cdot B_{\omega, \omega^{2}}^{-1}=\left(\frac{\operatorname{det} \square_{\omega}}{\operatorname{det} \square_{\mathcal{O}}}\right)^{-3} B_{\omega, \omega}^{-1} B_{\omega, \omega^{2}}^{-1}
$$

In string measure we need the following combination:

$$
\begin{aligned}
\left(\operatorname{det} \square{ }_{\mathcal{O}}\right)^{-5}\left(\operatorname{det} \square_{\omega^{3}}\right)^{-1} & =\left(\frac{\operatorname{det} \square_{\omega^{3}}}{\operatorname{det} \square_{\mathcal{O}}}\right)^{-1}\left(\frac{\operatorname{det} \square_{\omega}}{\operatorname{det} \square_{\mathcal{C}}}\right)^{-3} \\
& =B_{\omega, \omega} B_{\omega, \omega^{2}}
\end{aligned}
$$

Thus we obtain an expression for the string measure on moduli space in terms of holomorphic fields and their zeros. Moreover, we obtain some information about analytical properties of this string measure (a super-analog of the BelavinKnizhnik theorem). This information can be extracted from the analyticity of $R_{L, M}(s, \sigma)$ with respect to moduli.

This work is intimately connected with papers $[10,12]$. In [10] a super-analog of the so-called Mumford form was constructed. The Mumford superform yields (as its bosonic counterpart does) a measure on moduli space. Reference [10] contains an expression for this measure which is analogous to one suggested by Beilinson and Manin in the bosonic case [7]. The relation (5) was established in [12], where on this basis it was shown that the measure constructed in [10] coincides with the string measure. The results of [10] will be reproduced in [13] using a different language. The above considerations show that Eq. (5) and, hence, all the basic formulae of [12] follow from (1). Let us remark that some arguments in [12] (in particular, the formulation of an analog for the Belavin-Knizhnik theorem in terms of extended moduli superspace) employ the results of the present work.

This work is dedicated to the memory of Vadim Knizhnik whose talent evoked our profound admiration. His brilliant scientific activity resulted in great progress in resolution of many problems of theoretical physics and particularly in string theory.

\section{Superconformal Manifolds and the Fields on Them}

Let us consider a domain in (1|1)-dimensional complex superspace $\mathbb{C}^{1 / 1}$ and the operators of spinor derivatives acting on functions in this domain,

$$
\begin{aligned}
& D=\partial / \partial \zeta+\zeta \partial / \partial z, \\
& \bar{D}=\partial / \partial \bar{\zeta}+\bar{\zeta} \partial / \partial \bar{z}
\end{aligned}
$$


(from now on, $z$ denotes a complex bosonic coordinate, while $\zeta$ denotes a complex fermionic one). A transformation from coordinates $(z, \zeta)$ to coordinates $(\tilde{z}, \widetilde{\zeta})$ is called a superconformal transformation if it preserves the spinor derivatives up to a factor:

$$
\widetilde{\bar{D}}=F \cdot D, \quad \bar{D}=\bar{F} \cdot \bar{D} .
$$

Every superconformal transformation can be written in the following form:

$$
\begin{gathered}
\tilde{z}=u(z)-u^{\prime}(z) \varepsilon(z) \zeta, \\
\tilde{\zeta}=\sqrt{u^{\prime}(z)}\left(\zeta+\varepsilon(z)+\frac{1}{2} \varepsilon(z) \varepsilon^{\prime}(z) \zeta\right),
\end{gathered}
$$

where $u(z)$ [respectively $\varepsilon(z)]$ is a bosonic (respectively fermionic) holomorphic function.

A superconformal manifold is a manifold which is composed of (1|1)dimensional superdomains glued together by means of superconformal transformations. That is to say, a superconformal manifold can be covered by coordinate patches connected by (3). The underlying manifold, $\mathscr{N}_{\text {red }}$, for a superconformal manifold can be considered as a one-dimensional complex manifold provided with a spinor structure. Let us recall that to consider an underlying manifold amounts to discarding all the nilpotents. This reduction (when one discards all the nilpotents) is designated by the subscript red above. The underlying manifold is thus glued up by means of holomorphic transformations $\tilde{z}=u(z)$. The fact that the underlying manifold receives a spinor structure means that a choice of sign of $\sqrt{u^{\prime}(z)}$ is fixed consistently for every gluing. A supermanifold is said to be compact if its underlying manifold is compact. The genus of a superconformal manifold is defined as the genus of its underlying manifold.

To every one-dimensional complex manifold $N$ provided with a spinor structure one can assign a superconformal manifold, $\mathscr{N}$, with help of the following construction. If $N$ is glued up by means of transformations $\tilde{z}=u(z)$ then the superconformal manifold $\mathscr{N}$ is to be glued up by means of $z=u(z), \widetilde{\zeta}=\sqrt{u^{\prime}(z)} \zeta$. The resulting superconformal manifold can be called a superconformal manifold without odd (i.e. fermionic) parameters.

A field of type $(p, q)$ on a superconformal manifold is given by functions which are defined for every coordinate system and obey

$$
\tilde{\mathscr{A}}=F^{-2 p} \bar{F}^{-2 q} \mathscr{A}
$$

under superconformal coordinate changes. [Here $F$ is a factor entering Eq. (2).] Let us denote by $\mathscr{C}^{p, q}$ the space of type $(p, q)$ fields.

The operator $D$ can be regarded as an operator acting from $\mathscr{C}^{0, q}$ to $\mathscr{C}^{1 / 2, q}$. Analogously, $\bar{D}$ acts from $\mathscr{C}^{p, 0}$ to $\mathscr{C}^{p, 1 / 2}$. This defines holomorphic fields of type $(p, 0)$ as fields annihilated by this action of $\bar{D}$. If a holomorphic type $(1 / 2,0)$ field,

$$
\varphi(z, \zeta)=\alpha(z)+\zeta a(z),
$$

is defined in a simply connected domain, then it can be represented in the form $\varphi=D \psi$, where

$$
\psi(z, \zeta)=A(z)+\zeta \alpha(z)
$$


and $\partial A(z) / \partial z=a(z)$. The field $\psi$ is defined by the condition $D \psi=\varphi$ up to an additive constant. A field of type $(1 / 2,1 / 2)$ can be integrated over the superconformal manifold. Let us denote the integral of such a field $v$ over $\mathscr{N}$ as

$$
\int_{\mathscr{N}} \nu d^{2} z d^{2} \zeta
$$

Since the product of type $(1 / 2,0)$ and type $(0,1 / 2)$ fields is a field of type $(1 / 2,1 / 2)$ we can define an inner product of type $(1 / 2,0)$ fields:

$$
\left\langle\omega, \omega^{\prime}\right\rangle=\int \omega^{\prime} \bar{\omega} d^{2} z d^{2} \zeta .
$$

One can consider differential $(r, s)$-forms on a superconformal manifold, in the same way as on any complex manifold. [Remember that an $(r, s)$-form on a complex (super)domain with holomorphic coordinates $z_{i}$ (even and odd) is such a differential form which is a homogeneous polynomial of order $r(s)$ with respect to $\left.d z_{i}\left(d \bar{z}_{i}\right).\right]$ In the standard way one defines the operators $\partial$ which makes an $(r+1, s)$ form from an $(r, s)$-form, $\bar{\partial}$ which makes an $(r, s+1)$-form from an $(r, s)$-form, and the operator $d=\partial+\bar{\partial}$. For example, if $\omega=d z \cdot p+d \zeta \cdot \varrho$, then

$$
\begin{gathered}
\partial \omega=d \zeta d z\left(\partial_{\zeta} p-\partial_{z} \varrho\right)-d^{2} \zeta \partial_{\zeta} \varrho, \\
\bar{\partial} \omega=-d z d \bar{z} \partial_{\bar{z}} p-d \zeta d \bar{z} \partial_{\bar{z}} \varrho+d \bar{\zeta} d z \partial_{\bar{\xi}} p-d \zeta d \bar{\zeta} \partial_{\bar{\xi}} \varrho .
\end{gathered}
$$

A connection between the fields of types $(1 / 2,0),(0,1 / 2),(1 / 2,1 / 2)$ and the forms of types $(1,0),(0,1),(1,1)$ respectively will be of importance for what follows. To a field $\sigma$ of type $(1 / 2,0)$, let us assign a $(1,0)$-form $\Sigma=\alpha(\sigma)$ as follows:

$$
\sigma \stackrel{\alpha}{\mapsto} \Sigma=d \zeta \cdot \sigma+e \cdot D \sigma,
$$

where $e=d z-d \zeta \cdot \zeta$. Similarly, a field $\bar{\sigma}$ of type $(0,1 / 2)$ is connected with a $(0,1)$ form $\bar{\Sigma}=\bar{\alpha}(\bar{\sigma})$. It can be proved that these definitions are correct (that is, they do not depend on the coordinate choice). One has the following simple, but useful propositions.

If $\sigma$ is a holomorphic type $(1 / 2,0)$ field, then the corresponding $(1,0)$-form $\Sigma$ obeys $d \Sigma=0$ (i.e. it is closed). If $\sigma$ can be represented as $\sigma=D h$ with holomorphic $h$, then $\Sigma=d h$ (i.e. $\Sigma$ is exact). For an arbitrary field of type $(1 / 2,0)$ the form $\Sigma$ satisfies $\partial \Sigma=0$ (i.e. it is $\partial$-closed). Moreover, the map $\alpha$ is a one-to-one map of $\mathscr{C}^{1 / 2,0}$ onto the space of $\partial$-closed $(1,0)$-forms.

The proofs can be given in the following way. An arbitrary (1,0)-form $S$ can be written in coordinates $(z, \zeta)$ in the form $S=d \zeta \cdot \sigma+e \cdot p$. Taking the coefficient $\sigma$ in the term containing $d \zeta$ we obtain the map from the space of $(1,0)$-forms into $\mathscr{C}^{1 / 2,0}$. Let $\gamma$ denote this map. [To verify that $\sigma$ is a field of type $(1 / 2,0)$, one can use the relations $\tilde{e}=F^{2} e$ and $d \tilde{\zeta}=F^{-1} d \zeta+G e$, where $F$ is a function entering (2), and $G$ is some other function.] It is easy to check in local coordinates that there exists a unique $(1,0)$-form $\Sigma$ satisfying $\gamma(\Sigma)=\sigma, \partial \Sigma=0$, and that this form is given by Eq. (4). This immediately implies that the form $\Sigma$ is defined correctly by (4) and that the map $\alpha$ is one-to-one. Furthermore, if $\sigma$ is holomorphic, so is the form $\Sigma$, hence $\bar{\partial} \Sigma=0$. The last equation together with $\partial \Sigma=0$ implies $d \Sigma=0$.

Now let us assign to every type $(1 / 2,1 / 2)$ field $v$ a $(1,1)$-form $N=\beta(v)$ :

$$
v \stackrel{\beta}{\mapsto} N=d \zeta d \bar{\zeta} \cdot v+d \zeta \bar{e} \cdot \bar{D} v+d \bar{\zeta} e \cdot D v+e \bar{e} \cdot D \bar{D} v .
$$


It is easy to verify that this form is correctly defined. The construction of the form in Eq. (5) is connected with that in Eq. (4). Namely, if an odd holomorphic type $(1 / 2,0)$ field $\sigma_{1}$ is connected with a $(1,0)$-form $\Sigma_{1}=\alpha\left(\sigma_{1}\right)$, whereas an odd antiholomorphic type $(0,1 / 2)$ field $\bar{\sigma}_{2}$ is connected with a $(0,1)$-form $\bar{\Sigma}_{2}=\bar{\alpha}\left(\bar{\sigma}_{2}\right)$, then the type $(1 / 2,1 / 2)$ field $\bar{\sigma}_{2} \sigma_{1}$ is connected with $(1,1)$-form $\Sigma_{1} \wedge \bar{\Sigma}_{2}$. That is to say, $\beta\left(\bar{\sigma}_{2} \sigma_{1}\right)=\alpha\left(\sigma_{1}\right) \wedge \bar{\alpha}\left(\bar{\sigma}_{2}\right)$. One can check that a (1,1)-form $M=\beta(\mu)$ corresponding to a field $\mu$ of type $(1 / 2,1 / 2)$, satisfies $d M=0$. That is, $M$ is a closed 2-form, and hence it can be integrated over a (2|0)-dimensional submanifold of the considered superconformal manifold $\mathcal{N}$, the integral being unchanged under continuous deformations of this submanifold. Let us pick for this role such a closed (2|0)dimensional (real) submanifold $N$ which has the underlying manifold identical to that of $\mathscr{N}$. [There always exists a smooth (2|0)-dimensional submanifold $N$ in $\mathscr{N}$ with $N_{\text {red }}=\mathscr{N}_{\text {red }}$. On the other hand, one sometimes cannot choose such $N$ to be a complex analytic submanifold of $\mathcal{N}$.] Then we get the relation

$$
\int_{\mathcal{N}} \mu d^{2} z d^{2} \zeta=\frac{1}{2 i} \int_{N} \beta(\mu) .
$$

[The integral of the field $\mu$ over the superconformal manifold $\mathscr{N}$ coincides with the integral of the closed 2 -form $(2 i)^{-1} \beta(\mu)$ over the (2|0)-dimensional submanifold $N$.] The proof of this relation is obvious in the case when the field $\mu$ is non-zero in one coordinate chart only and the submanifold $N$ is defined in this chart by the equation $\zeta=\bar{\zeta}=0$. The general case can be obtained from the above by use of a partition of unity and by coordinate changes. Remember that if a manifold is covered by charts $\mathscr{U}_{i}$, one can always choose functions $f_{i}$ in such a way that $1=\sum f_{i}$ and that the support of $f_{i}$ is in $\mathscr{U}_{i}$. Then the proof of (6) amounts to the proof of this relation for the fields $\mu_{i}=f_{i} \mu$.

Later on we shall need also the following simple fact about $(2,0)$-forms. If a $(2,0)$-form $\Omega$ is $\partial$-closed, i.e. $\partial \Omega=0$, then it always is $\partial$-exact, i.e. it can be represented as $\Omega=\partial \tau$ for some globally defined $(1,0)$-form $\tau$. Indeed, if $\Omega$ is written in the form

$$
\Omega=d \zeta^{2} \cdot p+d \zeta e \cdot \varrho,
$$

then $\partial \Omega=0$ amounts to $\varrho=D p$. In this case we have $\Omega=\partial \tau$, where $\tau=e \cdot p$.

\section{Periods and Residues ${ }^{2}$}

Let us consider a holomorphic type $(1 / 2,0)$ field $\sigma$ on a superconformal manifold $\mathscr{N}$. The period of $\sigma$ with respect to a one-dimensional cycle $c$ in the underlying manifold can be defined as the integral of the (1,0)-form $\Sigma=\alpha(\sigma)$ over a closed (1|0)-dimensional (real) submanifold in $\mathscr{N}$, the underlying manifold of which coincides with the cycle $c$. Since the form $\Sigma$ is closed, the period so defined depends only on the homology class of $c$. If $\mathcal{N}$ is a compact superconformal manifold of genus $g$, all the periods of $\sigma$ are represented as linear combinations of its periods with respect to a standard basis $a^{1}, \ldots, a^{g}, b_{1}, \ldots, b_{g}$ in the one-dimensional

${ }^{2}$ Some results of this section have been independently obtained by A. M. Levin 
homology group of the underlying manifold of $\mathcal{N}$. Another definition of periods can be given with help of the universal covering, $\tilde{\mathscr{N}}$, of $\mathscr{N}$. The fundamental group of the underlying manifold of $\mathscr{N}$ acts on $\tilde{\mathcal{N}}$. For a field $\sigma$ on $\mathscr{N}$ there is a corresponding field $\tilde{\sigma}$ of type $(1 / 2,0)$ on $\tilde{\mathscr{N}}$. Since $\tilde{\mathscr{N}}$ is simply connected the latter field can be represented in the form $\tilde{\sigma}=D h$. The field $\tilde{\sigma}$ remains unchanged under the action of the fundamental group. Hence $h$ changes by an additive constant under each of such transformations. These constants are called the periods of $\sigma$ :

$$
\begin{aligned}
& \int_{a^{i}} \Sigma=h\left(\hat{a}^{i}(Z)\right)-h(Z), \\
& \int_{b_{i}} \Sigma=h\left(\hat{b}_{i}(Z)\right)-h(Z) .
\end{aligned}
$$

Here $\left\{\hat{a}^{i}, \hat{b}_{i}\right\}$ are the generators of the fundamental group, $\left\{a^{i}, b_{i}\right\}$ is the corresponding homology basis, $Z$ is a point in $\tilde{\mathscr{N}}$. Equation (7) follows from the relation $\tilde{\Sigma}=d h$, where $\tilde{\Sigma}$ is the lift of $\Sigma=\alpha(\sigma)$ to $\tilde{\Gamma}$.

One can prove a relation for periods of fields of type $(1 / 2,0)$ on a superconformal manifold generalizing the Riemann bilinear relations for differentials on a complex curve. The proof can be obtained by reducing to the case of ordinary two-dimensional manifolds. Namely, let $\omega, \omega^{\prime}$ be holomorphic type $(1 / 2,0)$ fields while $\Omega=\alpha(\omega)$ and $\Omega^{\prime}=\alpha\left(\omega^{\prime}\right)$ are the corresponding closed $(1,0)$-forms on a compact superconformal manifold $\mathscr{N}$ of genus g. Let $A^{l}, B_{j} ; A^{\prime i}, B_{j}^{\prime}$ $(i, j=1, \ldots, g)$ denote the periods of $\omega, \omega^{\prime}$ with respect to a standard homology basis $a^{i}, b_{j}$ in $\mathscr{N}_{\text {red }}$. Then, as we shall see shortly, one gets the following relations:

$$
\begin{gathered}
A^{i} B_{i}^{\prime}-B_{i} A^{\prime i}=0, \\
A^{\prime i} \bar{B}_{i}-B_{i}^{\prime} \bar{A}^{i}=2 i(-1)^{\tilde{\omega}^{\prime}}\left\langle\omega, \omega^{\prime}\right\rangle,
\end{gathered}
$$

where $\tilde{\omega}^{\prime}$ denotes the parity of $\omega^{\prime}$.

Indeed, consider a (2|0)-dimensional smooth submanifold $N$ in $\mathscr{N}$, such that $N_{\text {red }}=\mathscr{N}_{\text {red. }}$ On $N$, in the same way as on an ordinary two-dimensional closed manifold, one has then

$$
\int_{N} \Omega \wedge \Omega^{\prime}=A^{i} B_{i}^{\prime}-B_{i} A^{\prime i}
$$

On the other hand, a closed $(2,0)$-form, $\Omega \wedge \Omega^{\prime}$, is always exact on $\mathscr{N}$ (see the end of the preceding section). Therefore one has also

$$
\int_{N} \Omega \wedge \Omega^{\prime}=0
$$

thus proving Eq. (8). In a similar way, Eq. (6) together with the relation $\beta\left(\omega^{\prime} \bar{\omega}\right)$ $=(-1)^{\tilde{\omega}^{\prime}} \alpha\left(\omega^{\prime}\right) \wedge \overline{\alpha(\omega)}$ implies Eq. (9):

$$
\begin{aligned}
2 i\left\langle\omega, \omega^{\prime}\right\rangle & \equiv 2 i \int_{\mathcal{N}} \omega^{\prime} \bar{\omega} d^{2} z d^{2} \zeta=\int_{N} \beta\left(\omega^{\prime} \bar{\omega}\right) \\
& =(-1)^{\tilde{\omega}^{\prime}} \int_{N} \Omega^{\prime} \wedge \bar{\Omega}=(-1)^{\tilde{\omega}^{\prime}}\left(A^{\prime i} \bar{B}_{i}-B_{i}^{\prime} \bar{A}^{i}\right) .
\end{aligned}
$$

As we shall see later on, in a most important case (the normal case as it will be referred to) the dimension of the space of holomorphic type $(1 / 2,0)$ fields equals $(0 \mid g)$. Let $\mathscr{A}^{1 / 2}$ denote this space taken with inverse parity. [Thus 
$\mathscr{A}^{1 / 2}=\Pi H^{0}(\mathcal{N}, \omega)$ in the notation of Sect. 3; see also Appendix.] In the normal case $\operatorname{dim} \mathscr{A}^{1 / 2}=(g \mid 0)$ and the inner product in this space is non-degenerate. Therefore one can pick a basis $\omega_{1}, \ldots, \omega_{g}$ in $\mathscr{A}^{1 / 2}$, such that

$$
\int_{a^{2}} \alpha\left(\omega_{j}\right)=\delta_{j}^{i} .
$$

Equations (8), (9) imply, as in the usual case, that the period matrix,

$$
\tau_{i j}=\int_{b_{\imath}} \alpha\left(\omega_{j}\right)
$$

is symmetrical, $\tau_{i j}=\tau_{j i}$, and that

$$
\left\langle\omega_{i}, \omega_{j}\right\rangle=\operatorname{Im} \tau_{i j} .
$$

The definition of periodics can be used to generalize the definition of the Jacobian to the case of a compact superconformal manifold of genus $g$. The periods of holomorphic type $(1 / 2,0)$ fields with respect to a cycle $c$ define a linear functional on the space $\mathscr{A}^{1 / 2}$. The linear functionals corresponding to all possible cycles form a lattice $V$ in $\left(\mathscr{A}^{1 / 2}\right)^{*}$ called the period lattice. Let us define the Jacobi manifold (or the Jacobian) for a superconformal manifold $\mathscr{N}$ as a coset space of $\left(\mathscr{A}^{1 / 2}\right)^{*}$ with respect to $V$, i.e.

$$
J(\mathscr{N})=\left(\mathscr{A}^{1 / 2}\right)^{*} / V .
$$

In the normal case the Jacobian is a $g$-dimensional torus. The inner product in $\mathscr{A}^{1 / 2}$ defines a measure in it and, hence, in $J$. Let us prove that the volume of the Jacobian with respect to this measure equals 1 . For this aim let us consider a basis $\left\{a^{i}\right\}$ in $\left(\mathscr{A}^{1 / 2}\right)^{*}$ dual to the basis $\left\{\omega_{i}\right\}$ in $\mathscr{A}^{1 / 2}$ which has been defined by Eqs. (10)-(12) above. The inner product in $\left(\mathscr{A}^{1 / 2}\right)^{*}$ corresponding to that in $\mathscr{A}^{1 / 2}$ yields [cf. (12)]

$$
\left\langle a^{i}, a^{j}\right\rangle=(\operatorname{Im} \tau)^{-1 i j} .
$$

The period lattice, $V$, in $\left(\mathscr{A}^{1 / 2}\right)^{*}$ is generated (over the integers) by $2 g$ vectors $a^{i}, b_{i}$ $=\tau_{i j} a^{j}$. Now the volume of the Jacobian (13) equals the volume of the parallelepiped spanned by $\left\{a^{i}, b_{j}\right\}$ which, in turn, equals the determinant of the following $2 g \times 2 g$ matrix:

$$
G=\operatorname{Re}\left(\begin{array}{cc}
\left\langle a^{i}, a^{j}\right\rangle & \left\langle a^{i}, b_{l}\right\rangle \\
\left\langle b_{k}, a^{j}\right\rangle & \left\langle b_{k}, b_{l}\right\rangle
\end{array}\right)
$$

(Here Re appeared for we dealt with an hermitian inner product $\langle$,$\rangle .) Then we$ have (in matrix notation)

$$
\begin{aligned}
G & =\left(\begin{array}{cc}
(\operatorname{Im} \tau)^{-1} & (\operatorname{Im} \tau)^{-1} \cdot \operatorname{Re} \tau \\
\operatorname{Re} \tau \cdot(\operatorname{Im} \tau)^{-1} & \operatorname{Re} \tau \cdot(\operatorname{Im} \tau)^{-1} \cdot \operatorname{Re} \tau+\operatorname{Im} \tau
\end{array}\right) \\
& =\left(\begin{array}{cc}
(\operatorname{Im} \tau)^{-1} & 0 \\
\operatorname{Re} \tau \cdot(\operatorname{Im} \tau)^{-1} & 1
\end{array}\right) \cdot\left(\begin{array}{cc}
1 & \operatorname{Re} \tau \\
0 & \operatorname{Im} \tau
\end{array}\right),
\end{aligned}
$$

and, thus, (volume of $J$ ) $=\operatorname{det} G=1$. 
Using inner product in $\mathscr{A}^{1 / 2}$ one can, in the normal case, identify its dual, $\left(\mathscr{A}^{1 / 2}\right)^{*}$, with its complex conjugate, $\overline{\mathscr{A}^{1 / 2}}$. This allows one to consider the manifold $\bar{J}$ (i.e. Jacobi manifold with the complex structure conjugated) as a coset space of $\mathscr{A}^{1 / 2}$ by a lattice. (This will be used in [13].) More precisely, $\bar{J}$ can be regarded as a torus dual to the torus $J$.

Let us discuss now meromorphic functions on a superconformal manifold $\mathscr{N}$; a function $f$ on $\mathscr{N}$ is called meromorphic if locally it can be written in the form

$$
f=h / g,
$$

where $h, g$ are holomorphic, provided $g_{\text {red }}$ does not vanish identically. (Here $g_{\text {red }}$ denotes the numerical part of $g$ and can be regarded as a function of $\mathscr{N}_{\text {red }}$.) First of all, we note some simple properties of an even meromorphic function $f$ near a point where $f$ vanishes. Suppose that $f(P)=0$ and that $f_{\text {red }}$ has a simple zero at $P_{\text {red }} \in \mathscr{N}_{\text {red }}$. Then $f$ vanishes on a (0|1)-dimensional submanifold in $\mathscr{N}$ lying over $P_{\text {red }}$. It is easy to see that there exists a unique point $P_{0}$ in this submanifold, such that

$$
f\left(P_{0}\right)=0, \quad D f\left(P_{0}\right)=0
$$

(and, of course, $P_{0 \text { red }}=P_{\text {red }}$ ). Such a point $P_{0}$ will be called a principal simple zero of $f$. It is also easy to see that a function $f$ near its principal simple zero $P_{0}$ takes the form $\left(z-z_{0}-\zeta \zeta_{0}\right) u(z, \zeta)$, where $\left(z_{0}, \zeta_{0}\right)$ are the coordinates of $P_{0}$ and $u$ is a holomorphic function which does not vanish near $P_{0}$. [In this case, $f$ vanishes on the (0|1)-dimensional manifold $\left\{z-z_{0}-\zeta \zeta_{0}=0\right\}$ containing $\left.P_{0} \cdot\right]$

Now a function $f$ will be said to have a principal simple pole at $P_{0}^{\prime}$ if the function $f^{-1}$ has a principal simple zero at that point. These definitions can be extended in an obvious way to (even) meromorphic fields of type $(p, 0)$ (or to sections of line bundles to be considered later on). We have not made any definitions concerning the case when $f_{\text {red }}$ has multiple zeros or poles. This will be discussed later on in this paper, but for the time being it will not be needed.

Let us define the residue of a meromorphic type $(1 / 2,0)$ field $\omega$ at the point $p \in \mathscr{N}_{\text {red }}$ as $(2 \pi i)^{-1}$ times the period of this field with respect to a cycle encircling $p$. That is to say, if $\omega$ is represented in the form $(2 \pi i)^{-1} D h$ for a multivalued function $h$, then the residue equals to a change of $h$ when turning once around $p$. Equivalently, we can write

$$
\underset{p}{\operatorname{res} \omega}=\frac{1}{2 \pi i} \int_{C} \alpha(\omega)
$$

where $C$ is a closed loop [i.e. a (1|0)-dimensional submanifold with an obvious orientation] in $\mathcal{N}$, such that $C_{\text {red }}$ encircles $p$ (and provided there is inside $C_{\text {red }}$ no other point over which $\omega$ is singular). A meromorphic field $\omega$ can be locally represented as a power series

$$
\omega=\sum_{k=-n}^{+\infty}\left[\beta_{k}+\left(\zeta-\zeta_{0}\right) b_{k}\right]\left(z-z_{0}-\zeta \zeta_{0}\right)^{k}
$$

Then it is easy to check that the residue of $\omega$ at $p=\left(z_{0}, \zeta_{0}\right)_{\text {red }}$ equals $b_{-1}$ and that $b_{-1}$ depends only on the point $p \in \mathscr{N}_{\text {red }}$ and not on the choice of $\left(z_{0}, \zeta_{0}\right)$ over it. 
The sum of residues of a meromorphic type $(1 / 2,0)$ field $\omega$ equals zero, just as in the ordinary case. (Indeed, if $\mathscr{N}_{\text {red }}^{\prime}$ denotes the manifold $\mathscr{N}_{\text {red }}$ with all the points over which $\omega$ is singular removed, then the sum of cycles encircling all the singular points is homological to zero in $\mathscr{N}_{\text {red. }}^{\prime}$.)

We have considered above Riemann relations for holomorphic fields only. Analogous, though somewhat more complex, relations can be written also for meromorphic fields. In fact, let $\omega$ (respectively $\omega^{\prime}$ ) be a holomorphic (respectively meromorphic) odd field of type $(1 / 2,0)$ on $\mathscr{N}$. The periods of $\omega$ and $\omega^{\prime}$ with respect to cycles $a^{1}, \ldots, a^{g}, b_{1}, \ldots, b_{g}$ will be denoted by $A^{i}, B_{j}$ and $A^{\prime i}, B_{j}^{\prime}$ respectively. Let $C$ be a closed loop in $\mathscr{N}$ such that $C_{\text {red }}$ bounds a simply connected domain $\mathscr{D}_{\text {red }}$ in $\mathscr{N}_{\text {red }}$ (i.e. $C_{\text {red }}=\partial \mathscr{D}_{\text {red }}$ ) containing all the points over which $\omega^{\prime}$ is singular. Finally, let us represent $\omega$ in the form $\omega=D h$ in the superdomain $\mathscr{D}$ over $\mathscr{D}_{\text {red }}$. Here $h$ is a holomorphic function in $\mathscr{D}$ which can be chosen, for instance, as follows:

$$
h(P)=\int_{P_{0}}^{P} \alpha(\omega)
$$

for some fixed point $P_{0}$ in $\mathscr{D}$. Then we have

$$
A^{i} B_{i}^{\prime}-B_{i} A^{\prime i}=\int_{C} \alpha\left(h \omega^{\prime}\right) .
$$

One can verify this in a way analogous to the way of proving Eq. (8). Note that the ambiguity $h \rightarrow h+$ const does not matter in Eq. (18), for $\int_{C} \alpha\left(\omega^{\prime}\right)=0$ (i.e. the sum of residues of $\omega^{\prime}$ vanishes). Moreover,

$$
\int_{C} \alpha\left(h \omega^{\prime}\right)=2 \pi i \sum_{p} \underset{p}{\operatorname{res}}\left(h \omega^{\prime}\right),
$$

where the sum is over all the points $p \in \mathscr{N}_{\text {red }}$ over which $\omega^{\prime}$ is singular. Let us put (18) in a different form. For this aim we pick up a single point $P$ in $\mathscr{N}$ over each point $p$, that is $P_{\text {red }}=p$, and in a neighbourhood of each $P$ we choose local coordinates $Z=(z, \zeta)$ with the origin in $P$. Then

$$
\begin{aligned}
\omega(Z) & =\sum_{k=0}^{\infty}\left[\beta_{k}(P)+\zeta b_{k}(P)\right] z^{k}, \\
\omega^{\prime}(Z) & =\sum_{k=-n(P)}^{\infty}\left[\beta_{k}^{\prime}(P)+\zeta b_{k}^{\prime}(P)\right] z^{k} .
\end{aligned}
$$

In this case we have, by virtue of facts pointed out above,

$$
\begin{aligned}
A^{i} B_{i}^{\prime}-B_{i} A^{\prime i}= & 2 \pi i \sum_{P}\left\{\sum_{k=1}^{n(P)} \beta_{k-1}(P) \beta_{-k}^{\prime}(P)\right. \\
& \left.+\sum_{k=2}^{n(P)} \frac{b_{k-2}(P) b_{-k}^{\prime}(P)}{k-1}+b_{-1}^{\prime}(P) \int_{P_{0}}^{P} \alpha(\omega)\right\} .
\end{aligned}
$$

(Note that, in particular, $\underset{p}{\operatorname{res} \omega^{\prime}}=b_{-1}^{\prime}(P)$ and $\sum_{P} b_{-1}^{\prime}(P)=0$.)

By the same methods one can prove that a super analog of Weil coupling is symmetric. (It plays an important role in computing the determinants of Laplacians on superconformal manifolds.) Let $\varphi, \varphi^{\prime}$ be even meromorphic 
functions on $\mathscr{N}$ satisfying the following restrictions. All the zeros and poles of $\varphi, \varphi^{\prime}$ are simple and principal and the set of zeros and poles of $\varphi_{\text {red }}$ does not intersect with that of $\varphi_{\text {red }}^{\prime}$. Let us denote by $N_{k}, P_{k}$ the points of principal zeros and poles of $\varphi$ and by $N_{n}^{\prime}, P_{n}^{\prime}$ analogous points for $\varphi^{\prime}$. The coupling of $\varphi$ and $\varphi^{\prime}$ is defined as follows:

$$
\left[\varphi^{\prime}, \varphi\right]=\prod_{k} \varphi^{\prime}\left(N_{k}\right) / \varphi^{\prime}\left(P_{k}\right) .
$$

(An extension of this definition to arbitrary meromorphic functions $\varphi, \varphi^{\prime}$ with still nonintersecting sets of zeros and poles of $\varphi_{\text {red }}$ and $\varphi_{\text {red }}^{\prime}$ can be obtained, in particular, by means of expressions for $\left[\varphi^{\prime}, \varphi\right]$ used below.)

Let us prove that

$$
\left[\varphi, \varphi^{\prime}\right]=\left[\varphi^{\prime}, \varphi\right] .
$$

For this aim let us pick two closed loops $C, C^{\prime}$ in $\mathscr{N}$ such that $C_{\text {red }}$ bounds a simply connected domain $\mathscr{U}_{\text {red }}\left(C_{\text {red }}=\partial \mathscr{U}_{\text {red }}\right)$ in $\mathscr{N}_{\text {red }}$, which contains all the points $\left(N_{k}\right)_{\text {red }}$, $\left(P_{k}\right)_{\text {red }}$; and similarly for $C_{\text {red. }}^{\prime}$. Then using a relation analogous to Eq. (18) we find:

$$
\begin{aligned}
\log & {\left[\varphi^{\prime}, \varphi\right]-\log \left[\varphi, \varphi^{\prime}\right] } \\
= & \sum_{k}\left[\underset{\left(N_{k}\right)_{\mathrm{red}}}{\operatorname{res}}\left(\log \varphi^{\prime} D \log \varphi\right)+\underset{\left(P_{k}\right)_{\mathrm{red}}}{\operatorname{res}}\left(\log \varphi^{\prime} D \log \varphi\right)\right] \\
& -\sum_{n}\left[\underset{\left(N_{n}^{\prime}\right)_{\mathrm{red}}}{\operatorname{res}}\left(\log \varphi D \log \varphi^{\prime}\right)+\underset{\left(P_{n}^{\prime}\right)_{\mathrm{red}}}{\operatorname{res}}\left(\log \varphi D \log \varphi^{\prime}\right)\right] \\
= & \int_{C} \alpha\left(\log \varphi^{\prime} D \log \varphi\right)-\int_{C^{\prime}} \alpha\left(\log \varphi D \log \varphi^{\prime}\right) \\
= & A^{\prime i} B_{i}-B_{i}^{\prime} A^{i}=0 \bmod (2 \pi i \mathbb{Z}),
\end{aligned}
$$

where $A^{i}, B_{j}$ (respectively $A^{\prime i}, B_{j}^{\prime}$ ) are the periods of $D \log \varphi\left(\right.$ respectively $\left.D \log \varphi^{\prime}\right)$; each of these, however, always being $2 \pi i$ times an integer, hence, the last equality in (24). We have also used here, that $\log \varphi$ is singlevalued near $C^{\prime}$ (and, analogously, is $\log \varphi^{\prime}$ near $\left.C\right)$ and that a residue $\operatorname{res}(f D \log \varphi)$ equals $f(P)$ if $\varphi$ has a principal simple zero at $P$, and it equals $-f(P)$ if $\varphi$ has a principal simple pole at $P$. (This can be proved by a direct verification of definitions.)

\section{Line Bundles. Cohomology}

A line bundle $L$ over a complex supermanifold is called a holomorphic bundle if it is glued up from trivial bundles with the fibre $\mathbb{C}^{1 / 0}$ by means of holomorphic transformations. These transformations must have the following form:

$$
(Z, l) \sim(\tilde{Z}, \tilde{l})=(f(Z), g(Z) l),
$$

where $Z, \tilde{Z}$ are local coordinates in the base; $l, \tilde{l}$ are coordinates in $\mathbb{C}^{1 \mid 0} ; f, g$ are holomorphic functions. The functions $g$ are called transition functions of the bundle $L$. A section of such a line bundle is specified by a set of functions $h$ given in local coordinate charts and related by transformations $\tilde{h}(\tilde{Z})=g(Z) h(Z)$. Let us define the holomorphic bundle $\omega$ on a superconformal manifold as a bundle glued 
up by means of transformations

$$
(Z, l) \sim(\tilde{Z}, \tilde{l})=\left(f(Z), F^{-1}(Z) l\right),
$$

where $f$ is a superconformal transformation and $F$ is the corresponding conformal factor defined by the relation $\widetilde{D}=F D$ (cf. Sect. 1). Then the fields of type $(p, q)$ considered in Sect. 1 can be regarded as sections of the bundle $\omega^{p} \bar{\omega}^{q}$, the tensor product of $p$ copies of $\omega$ times $q$ copies of the antiholomorphic bundle $\bar{\omega}$ (complex conjugate of $\omega$ ).

Let us denote by $\mathscr{E}^{0, k}(L)$ the space of $(0, k)$-forms taking values in a line bundle $L$ on a complex supermanifold $\mathscr{N}$. If $L$ is a holomorphic line bundle, then we have a well-defined operator $\bar{\partial}$ acting from $\mathscr{E}^{0, k}(L)$ to $\mathscr{E}^{0, k+1}(L)$. Using this operator one defines in a usual manner the cohomology groups $H^{k}(\mathcal{N}, L)$ as $\bar{\partial}$-closed $(0, k)$-forms modulo $\bar{\partial}$-exact forms. Then $H^{0}(\mathcal{N}, L)$, in particular, coincides with the space $\mathscr{A}(L)$ of holomorphic sections of $L$. If, moreover, $\mathscr{N}$ is a superconformal manifold, one can describe $H^{0}(\mathscr{N}, L)$ also as the space of sections $\varphi$ of $L$ satisfying the condition $\bar{D} \varphi=0$. (This follows from $\bar{D}^{2}=\partial / \partial \bar{z}$.) Then the group $H^{1}(\mathcal{N}, L)$ can be described as the space of fields of type $(0,1 / 2)$ with values in $L$ (i.e. sections of $L \otimes \bar{\omega})$ modulo the image of the operator $\bar{D}$. [Since $L$ is a holomorphic bundle we have a welldefined operator $\bar{D}$ acting from the space, $\Gamma(L)$, of sections of $L$ to the space, $\Gamma(L \otimes \bar{\omega})$, of sections of $L \otimes \bar{\omega}$.] In order to verify that this description of $H^{1}(\mathcal{N}, L)$ is correct, one must observe that the correspondence between fields of type $(0,1 / 2)$ and $(0,1)$-forms (see Sect. 1) can be extended to fields taking values in $L$. [Every type $(0,1 / 2)$ field $\bar{\sigma}$ with values in $L$ is related to a $\bar{\partial}$-closed $(0,1)$-form $\bar{\Sigma}=d \bar{\zeta} \bar{\sigma}+\bar{e} \bar{D} \bar{\sigma}$ with values in $L$; if $\bar{\sigma}=\bar{D} \varphi$, then $\bar{\Sigma}=\bar{\partial} \varphi$; and vice versa.] Furthermore, one can check that $H^{k}(\mathcal{N}, L)=0$ for $k \geqq 2$. (We shall not use this and therefore omit the proof.)

Let us show now that the space $H^{1}(\mathscr{N}, L)$ can be identified with the space of linear functionals on $H^{0}\left(\mathscr{N}, L^{*} \otimes \omega\right)=\mathscr{A}\left(L^{*} \otimes \omega\right)$; namely,

$$
H^{1}(\mathscr{N}, L)=\Pi H^{0}\left(\mathscr{N}^{*}, L^{*} \otimes \omega\right)^{*} .
$$

Here $\Pi$ indicates that this identification relates the elements with opposite parities. [To say more on the notation, if $E$ is a linear space, then $E^{*}$ denotes its dual, i.e. the space of linear functionals on $E$; if $L$ is a line bundle, $L^{*}$ is the dual line bundle; and finally, if $A$ is a linear operator acting from $E_{1}$ to $E_{2}$, then $A^{*}$ is the (dual) conjugated linear operator which acts from $E_{2}^{*}$ to $E_{1}^{*}$.] Now, we can define a scalar product of a section of $L^{*} \otimes \omega$ and a section of $L \otimes \bar{\omega}$, since sections of the bundle $\omega \otimes \bar{\omega}$ [i.e. the fields of type $(1 / 2,1 / 2)]$ can be integrated over $\mathscr{T}$. This scalar product allows us to identify $\Gamma(L \otimes \bar{\omega})^{*}$ with $\Gamma\left(L^{*} \otimes \omega\right)$. In a similar way, $\Gamma(L)^{*}$ can be identified with $\Gamma\left(L^{*} \otimes \omega \otimes \bar{\omega}\right)$. Let us denote by $\bar{D}_{L}$ the $\bar{D}$-operator that acts from $\Gamma(L)$ to $\Gamma(L \otimes \bar{\omega})$. Its conjugate, $\bar{D}_{L}^{*}$, can be viewed on as an operator acting from $\Gamma\left(L^{*} \otimes \omega\right)=\Gamma(L \otimes \bar{\omega})^{*}$ to $\Gamma\left(L^{*} \otimes \bar{\omega} \otimes \omega\right)=\Gamma(L)^{*}$. With help of integrating by parts, one sees that $\bar{D}_{L}^{*}$ coincides up to a sign with $\bar{D}_{L^{*} \otimes \omega}$. This allows $H^{1}(\mathcal{N}, L)$ (i.e. the cokernel of $\left.\bar{D}_{L}\right)$ to be identified with $H^{0}\left(\cdot \mathcal{N}, L^{*} \otimes \omega\right)^{*}$ (i.e. the space dual to the kernel of $\bar{D}_{L^{*} \otimes \omega}$ ). Since the operator $\bar{D}_{L}$ changes the parity, one gets the opposite parities of elements identified in both spaces, hence, $\Pi$ in Eq. (27).

Besides the constructions of the group $H^{1}(\mathcal{N}, L)$ described above it is often convenient to make use also of the following construction. Let us fix a covering of 
$\mathscr{N}$ by open superdomains $\mathscr{U}_{1}, \ldots, \mathscr{U}_{n}$. It can be proved that the result of the construction below doesn't depend on the choice of covering $\left\{\mathscr{U}_{i}\right\}$, provided, for instance, $\mathscr{U}_{i} \neq \mathscr{N}$ for all $i$. A 0 -cochain (in the sense of Čech) is defined as a set of holomorphic sections, $\varphi_{1}, \ldots, \varphi_{n}$, of $L$ over the domains $\mathscr{U}_{1}, \ldots, \mathscr{U}_{n}$. A 1 -cochain is a set of holomorphic sections $\varphi_{i j}$ over the domains $\mathscr{U}_{i} \cap \mathscr{U}_{j}$ satisfying $\varphi_{i j}=-\varphi_{j i}$. A 1 -cochain is called a cocycle if

$$
\varphi_{i j}+\varphi_{j k}+\varphi_{k i}=0
$$

on $\mathscr{U}_{i} \cap \mathscr{U}_{j} \cap \mathscr{U}_{k}$. A 1-cocycle is said to be cohomologous to zero if it can be represented in the form $\varphi_{i j}=\psi_{i}-\psi_{j}$ for a 0 -cochain $\psi_{i}$. The group $H^{1}(\mathscr{N}, L)$ (in the sense of (̌ech) is defined as the quotient of the group of 1-cocycles by the subgroup of 1-cocycles cohomologous to zero. The equivalence of the last definition and the definitions above follows easily from the elementary sheaf theory. Without going into details of the proof let us show only how a type $(0,1 / 2)$ field $\bar{\varrho}$ defines a 1 -cocycle in the sense of $\breve{C}$ ech. Pick in every domain $\mathscr{U}_{i}$ a solution, $\varphi_{i}$, to the equation (here we have to require each $\mathscr{U}_{i}$ to be simply connected)

$$
\bar{D} \varphi+\bar{\varrho}=0 \text {. }
$$

This equation may have no solution on the whole of $\mathscr{N}$, but in $\mathscr{U}_{i}$ it always has solutions and any two of such solutions differ by a holomorphic function. Define a Čech 1 -cocycle by taking $f_{i j}=\varphi_{i}-\varphi_{j}$. In the case when $\varrho=\bar{D} \alpha$, the equation (29) has a solution on $\mathscr{N}$ and $f_{i j}$ is cohomologous to zero. We find that the group $H^{1}\left(\mathscr{N}^{\prime}, L\right)$ defined firstly is mapped into Čech's $H^{1}(\mathscr{N}, L)$. This map happens, in fact, to be an isomorphism.

Let us use the last description of $H^{1}(\mathscr{N}, L)$ to find the tangent space of the superconformal moduli space $\mathscr{M}_{g}$, that is to the space of equivalence classes of superconformal manifolds of genus $g$. Remember that a superconformal manifold has been viewed on as a manifold glued up from (1|1)-dimensional complex superdomains $\mathscr{U}_{i}$ by means of superconformal transformations. In order to describe an infinitesimal variation of this superconformal manifold, one has to consider an infinitesimal variation of transformations which glue $\mathscr{U}_{i}$ and $\mathscr{U}_{j}$ together. Every such variation is given by an infinitesimal superconformal transformation of $\mathscr{U}_{i} \cap \mathscr{U}_{j}$, that is it is given by a superconformal vector field on $\mathscr{U}_{i} \cap \mathscr{U}_{j}$. Such a field can be represented by a holomorphic even field $v_{i j}$ of type $(-1,0)$ on $\mathscr{U}_{i} \cap \mathscr{U}_{j}$. [A field $v(z, \zeta)$ is related to an infinitesimal superconformal transformation $\delta z=v-\frac{1}{2} \zeta D v, \delta \zeta=\frac{1}{2} D v$.] The set of fields $v_{i j}$ can be thought of as a 1 -cocycle defining a cohomology class $v \in H^{1}\left(\mathscr{N}, \omega^{-2}\right)$. If the fields $v_{i j}, v_{i j}^{\prime}$ define the same cohomology class, that is $v_{i j}^{\prime}-v_{i j}=v_{i}-v_{j}$ for holomorphic type $(-1,0)$ fields $v_{i}$ on $\mathscr{U}_{i}$, then the corresponding variations of our superconformal manifold are equivalent. (Superconformal transformations of $\mathscr{U}_{i}$ defined by the fields $v_{i}$ just give the required equivalence.) Thus we can identify the tangent space to the moduli space $\mathscr{M}_{g}$ with $H^{1}\left(\mathscr{N}, \omega^{-2}\right)$. By virtue of the duality (27) the cotangent space of $\mathscr{M}_{g}$ (i.e. dual of the tangent space) gets identified with $\Pi H^{0}\left(\mathscr{N}, \omega^{3}\right)$. As we shall see later on (Sect. 5), if $g>1$ then $H^{0}\left(\mathscr{N}, \omega^{3}\right)$ is a $(2 g-2 \mid 3 g-3)$-dimensional complex superspace. Therefore, $\operatorname{dim}_{\mathbb{C}} \mathscr{M}_{g}=(3 g-3 \mid 2 g-2)$. [Since $\Pi H^{0}\left(\mathscr{N}, \omega^{3}\right)$ is a complex linear manifold, $\mathscr{M}_{g}$ becomes naturally a complex supermanifold.] 


\section{Classification of Line Bundles}

A formal linear combination of a finite number of points in a superconformal manifold $\mathscr{N}$ with integer coefficients will be called a divisor on $\mathscr{N}$. The set of all divisors forms an abelian group with respect to an obvious addition law. A divisor, say $\sum n_{i} P_{i}$, is said to be linearly equivalent to zero if there exists an even meromorphic function $f$ on $\mathscr{N}$ satisfying the following conditions. Let $\mathscr{U}$ be a superdomain in $\mathscr{N}$ sufficiently small for a local coordinate system $(z, \zeta)$ covering $\mathscr{U}$ to exist. If $\mathscr{U}$ contains no points of the divisor in view, then $f$ must be holomorphic in $\mathscr{U}$ and $f_{\text {red }}$ must have no zeros in $\mathscr{U}_{\text {red }}$.

Suppose now that some points, $P_{i_{1}}, \ldots, P_{i_{K}}$, of the divisor are contained in $\mathscr{U}$. Then $f$ must take in $\mathscr{U}$ the following form:

$$
f=h \prod_{k=1}^{K}\left(z-z_{k}-\zeta \zeta_{k}\right)^{n_{{ }}},
$$

where $\left(z_{k}, \zeta_{k}\right)$ are coordinates of $P_{i_{k}}$ in $\mathscr{U}$, and $h$ is a holomorphic function in $\mathscr{U}$, such that $h_{\text {red }}$ has no zeros in $\mathscr{U}_{\text {red }}$. For example, if $f$ is a meromorphic function on $\mathscr{N}$ and has only principal simple zeros $N_{i}$ and principal simple poles $P_{i}$, then $f$ corresponds to the divisor $\sum N_{i}-\sum P_{i}$ which is thus linear equivalent to zero. It must be pointed out, however, that in general two different divisors, each being linearly equivalent to zero, may be related to the same meromorphic function. This follows from the fact that expansion (30) may be ambiguous. In fact, if $a$ is an even constant satisfying $a^{2}=0$, then, for instance, the following function can be expanded in two different ways: $z(z+2 a)=(z+a)^{2}$. On the other hand, we notice that not every possible meromorphic function on $\mathscr{N}$ corresponds to some divisor. [If $f_{\text {red }}$ has, say, a multiple zero at $P_{\text {red }} \in \mathscr{U}_{\text {red }}$, then it is possible that, nevertheless, $f$ cannot be represented in $\mathscr{U}$ in the form (30).] There exists however an alternative definition of divisors which allows one to consider arbitrary meromorphic functions $f$ with $f_{\text {red }} \neq 0$ (see the Appendix).

Now, two divisors are said to be linear equivalent if their difference is linear equivalent to zero. The group of classes of linear equivalent divisors on $\mathscr{N}$ will be denoted by $\operatorname{Cl}(\mathscr{N})$. Let us show that every holomorphic line bundle on $\mathscr{N}$ can be related to some element of $C l(\mathcal{N})$, so that isomorphic bundles correspond to the same element. Conversely, every element of $\mathrm{Cl}(\mathscr{N})$ will correspond to some isomorphism class of holomorphic line bundles. Indeed, let us consider a holomorphic line bundle $L$ and pick a meromorphic section $s$ of $L$ which possesses only principal simple zeros and poles. (Thus, in particular $s_{\text {red }}$, a section of $L_{\text {red }}$ on $\mathscr{N}_{\text {red }}$, must have only simple zeros and poles. The definition of a principal simple zero for sections of $L$ is in fact the same as for meromorphic functions; it is defined by the conditions $s=0, D s=0$. A principal simple pole of $s$ is defined also as a principal simple zero of $s^{-1}$, a section of the dual bundle $L^{*}$.) The divisor corresponding to such a section $s$ is defined as $\sum N_{i}-\sum P_{j}$, where $N_{i}\left(P_{j}\right)$ are principal simple zeros (poles) of $s$. If $s_{1}, s_{2}$ are two sections obeying the above constraint, then their divisors differ by a divisor related to the meromorphic function $s_{1} / s_{2}$. Thus we see that every holomorphic line bundle is related to a definite divisor class in $C l(\mathcal{N})$. Conversely, if a divisor is of the form $P$, for a single point $P$ in $\mathscr{N}$, then it is related to a line bundle $\mathcal{O}(P)$ defined in the following way. 
Let $Z=(z, \zeta)$ be coordinates in a superdomain $\mathscr{U}_{1}$ containing $P=\left(z_{0}, \zeta_{0}\right)$ and $\mathscr{U}_{2}$ be a second domain not containing $P$ such that $\mathscr{U}_{1}, \mathscr{U}_{2}$ cover $\mathscr{N}$. Then $\mathscr{O}(P)$ can be glued up from trivial bundles over $\mathscr{U}_{1}$ and $\mathscr{U}_{2}$ by means of the transformation over $\mathscr{U}_{1} \cap \mathscr{U}_{2}$,

$$
(Z, l) \sim(\widetilde{Z}, \tilde{l})=\left(f(Z),\left(z-z_{0}-\zeta \zeta_{0}\right)^{-1} l\right) .
$$

The bundle $\mathcal{O}(P)$, so defined, possesses a holomorphic section given by the functions $\widetilde{l}(\tilde{Z})=1$ in $\mathscr{U}_{2}$ and $l(Z)=z-z_{0}-\zeta \zeta_{0}$ in $\mathscr{U}_{1}$. The divisor of this section equals $P$, as required. In general, a divisor $\sum n_{i} P_{i}$ corresponds to a bundle defined as the tensor product $\bigotimes_{i} \mathcal{O}\left(P_{i}\right)^{n_{t}}$. [Here $L^{n_{i}}$ for $n_{i}<0$ means $\left(L^{*}\right)^{-n_{t}}$.] It is easy to verify that the bundle corresponding to a divisor depends up to isomorphism only on its class and that we get thus a one-to-one correspondence between the group $C l(\mathscr{N})$ and isomorphism classes of holomorphic line bundles on $\mathscr{N}$.

The integer $d=\sum n_{i}$ is called the degree, $d=\operatorname{deg} \mathscr{D}$, of a divisor $\mathscr{D}=\sum n_{i} P_{i}$. The degree of a divisor equals $\operatorname{deg} L$, the topological number of the corresponding line bundle $L$ on $\mathscr{N}$, as one can readily see by descending to $\mathscr{N}_{\text {red }}(\operatorname{deg} L$ is defined simply as deg $L_{\text {red. }}$.) In particular, for a divisor $\mathscr{D}$ linearly equivalent to zero one gets $\operatorname{deg} \mathscr{D}=0$, as one can also check directly by considering the divisor of $f_{\text {red }}$ on $\mathscr{N}_{\text {red }}$, where $f$ is a meromorphic function corresponding to $\mathscr{D}$. Now holomorphic line bundles of degree zero (i.e. topologically trivial holomorphic bundles) are described by the superspace $C l_{0}(\mathscr{N})$ of classes of divisors of zero degree.

If $L$ is a topologically trivial bundle, that is a bundle topologically isomorphic to the direct product $\mathscr{N} \times \mathbb{C}^{1 \mid 0}$, then the $\bar{\partial}$-operator in this bundle can be written in the form $\bar{\partial}_{L}=\bar{\partial}+A$, where $\bar{\partial}$ corresponds to a holomorphically trivial bundle (i.e. it acts on functions in the standard way) and $A$ is an even $\bar{\partial}$-closed $(0,1)$-form on $\mathscr{N}$. Conversely, every $\bar{\partial}$-closed $(0,1)$-form on $\mathscr{N}$ corresponds to a topologically trivial holomorphic bundle. If $u$ is an even (regular) invertible function (i.e. $u_{\text {red }}$ nowhere vanishes), then two $(0,1)$-forms $A^{\prime}$ and $A$, connected by the relation

$$
A^{\prime}=A+u^{-1} \bar{\partial} u
$$

correspond to isomorphic bundles. [This follows from $\bar{\partial}+A^{\prime}=\hat{u}^{-1}(\bar{\partial}+A) \hat{u}$, where $\hat{u}$ is the operator of multiplication by $u$ which sets up the isomorphism of bundles.] If $u=\exp \lambda$, Eq. (31) takes the form $A^{\prime}=A+\bar{\partial} \lambda$. The latter means that $A^{\prime}$ and $A$ generate the same even element in $H^{1}(\mathcal{N}, \mathcal{O})$ where $\mathcal{O}$ is the trivial bundle. Consequently, the superspace of classes (i.e. moduli space) of topologically trivial holomorphic line bundles can be represented as a quotient of $H^{1}(\mathcal{N}, \mathcal{O})$ with respect to an additional equivalence [for the cohomological equivalence $A \sim A$ $+\bar{\partial} \lambda$ doesn't include (31) in the case when $\log u$ cannot be made single valued].

We could get the same result by dealing with the $\bar{D}$-operator in $L$, that is $\bar{D}_{L}=\bar{D}+\bar{\varrho}$, where $\bar{\varrho}$ is an odd field of type $(0,1 / 2)$ on $\mathscr{N}$. Analogously to Eq. (31), $\varrho$ and $\bar{\varrho}^{\prime}$ correspond to isomorphic bundles iff

$$
\bar{\varrho}^{\prime}=\bar{Q}+u^{-1} \bar{D} u
$$

for an invertible function $u$. In particular, we come again (cf. the last section) to an equivalent description of $H^{1}(\mathscr{N}, \mathcal{O})$ as the space of classes of type $(0,1 / 2)$ fields modulo the fields of the form $\bar{D} \lambda$. Similarly, the moduli space of topologically 
trivial line bundles gets a description as the space of cohomology classes from $H^{1}(\mathscr{N}, \mathcal{O})$ modulo the classes of the fields $u^{-1} \bar{D} u$. Further, $H^{1}(\mathscr{N}, \mathcal{O})$ can be identified according to Eq. (27) with $\left(\mathscr{A}^{1 / 2}\right)^{*}$, for $\mathscr{A}^{1 / 2}=\Pi H^{0}(\mathscr{N}, \omega)$, the space of holomorphic type $(1 / 2,0)$ fields on $\mathscr{N}$. Thus our moduli space of bundles gets identified with a quotient of $\left(\mathscr{A}^{1 / 2}\right)^{*}$ by a subgroup. Let us show that this subgroup coincides with the period lattice $V$ in $\left(\mathscr{A}^{1 / 2}\right)^{*}$. For this aim, we must prove that the linear functionals on $\mathscr{A}^{1 / 2}$,

$$
\mathscr{A}^{1 / 2} \ni \sigma \mapsto(\bar{\varrho}, \sigma) \equiv \frac{1}{\pi} \int_{\mathscr{N}} \bar{\varrho} \sigma d^{2} z d^{2} \zeta
$$

[which set up the equivalence $H^{1}(\mathscr{N}, \mathcal{O})=\left(\mathscr{A}^{1 / 2}\right)^{*}$ ] belong to the lattice $V$ if $\bar{\varrho}=u^{-1} \bar{D} u$ for an invertible $u$. Using the methods of Sect. 2 [cf. Eq. (6)], we choose a (2|0)-dimensional submanifold $N$ in $\mathcal{N}$, so that

$$
\left(u^{-1} \bar{D} u, \sigma\right)=\frac{1}{\pi} \int_{\mathscr{N}} u^{-1} \bar{D} u \cdot \sigma d^{2} z d^{2} \zeta=\frac{1}{2 \pi i} \int_{N} \beta\left(u^{-1} \bar{D} u \cdot \sigma\right)
$$

Then, noticing that $\beta\left(u^{-1} \bar{D} u \cdot \sigma\right)$ can be replaced in the integral by $\alpha(\sigma) \wedge u^{-1} d u$, for they differ by an exact 2-form, as one can check directly [namely, by $-d\left(e \cdot u^{-1} D u \cdot \sigma\right)$, where $e=d z+\zeta d \zeta$ as before], we find

$$
\left(u^{-1} \bar{D} u, \sigma\right)=\frac{1}{2 \pi i} \int_{N} \alpha(\sigma) \wedge u^{-1} d u=\frac{1}{2 \pi i}\left(A^{i} B_{i}^{\prime}-B_{i} A^{\prime i}\right),
$$

where $A^{i}, B_{j}$ are the periods of $\alpha(\sigma)$ and $A^{\prime i}, B_{j}^{\prime}$ are the periods of $u^{-1} d u$. Since all the periods of the latter 1 -form, $u^{-1} d u$, are integer (times $2 \pi i$ ), we conclude that the linear functional $\sigma \mapsto\left(u^{-1} \bar{D} u, \sigma\right)$ belongs to the period lattice $V$ as desired. Thus we have seen that the moduli space of topologically trivial holomorphic line bundles is isomorphic to the Jacobian $J(\mathscr{N})=\left(\mathscr{A}^{1 / 2}\right)^{*} / V$.

Now we have two different descriptions of the above moduli space of bundles: in terms of $C l_{0}(\mathscr{N})$ and in terms of $J(\mathscr{N})$. Therefore $C l_{0}(\mathscr{N})$ and $J(\mathscr{N})$ must be isomorphic. One can find this isomorphism explicitly. Let us first construct a map $C l_{0}(\mathscr{N}) \rightarrow J(\mathscr{N})$. Let $\mathscr{D}=\sum_{i=1}^{N} n_{i} P_{i}$ be a divisor of zero degree; $\sum n_{i}=0$. Let us fix some point $P_{0}$ in $\mathscr{N}$ and connect it by (1|0)-dimensional real submanifolds $C_{1}, \ldots, C_{N}$ with the points $P_{1}, \ldots, P_{N}$. Then this divisor yields a linear functional on $\mathscr{A}^{1 / 2}$ as follows:

$$
\mathscr{A}^{1 / 2} \ni \sigma \mapsto \sum n_{i} \int_{C_{\imath}} \alpha(\sigma)
$$

Under a change of curves $C_{i}$ this functional may change only by an element of the period lattice $V$. Hence, every divisor $\mathscr{D}$ with $\operatorname{deg} \mathscr{D}=0$ defines a unique point in $J(\mathscr{N})=\left(\mathscr{A}^{1 / 2}\right)^{*} / V$. (Note that this doesn't depend also on the choice of $P_{0}$ by virtue of the condition $\sum n_{i}=0$.) Moreover, one can see that divisors linearly equivalent to zero are mapped into zero of $J(\mathscr{N})$. Thus, we get a map $C l_{0}(\mathscr{N}) \rightarrow J(\mathscr{N})$. In fact, let $\mathscr{U}_{1}$ be a superdomain containing all the points $P_{1}, \ldots, P_{N}$, such that $\mathscr{U}_{1 \text { red }}$ is simply connected, let $C$ be a closed loop in $\mathscr{N}$ such that $C_{\text {red }}=\partial \mathscr{U}_{1 \text { red }}$ and let $\mathscr{U}_{2}$ be the rest of $\mathscr{N}$. Then the functional (34) can be rewritten modulo $V$ as follows:

$$
\sigma \mapsto \mathscr{D}(\sigma)=\sum_{i=1}^{N} n_{i} h\left(P_{i}\right)=\frac{1}{2 \pi i} \int_{C} \alpha\left(h \varphi^{-1} D \varphi\right),
$$


where $\sigma \in \mathscr{A}^{1 / 2}, \mathscr{D}=\sum n_{i} P_{i}, \operatorname{deg} \mathscr{D}=0, h$ is a holomorphic function in $\mathscr{U}_{1}$ with $D h=\sigma$, and $\varphi$ is an arbitrary meromorphic function in $\mathscr{U}_{1}$ corresponding to $\mathscr{D}$. [If, for instance, $\mathscr{U}_{1}$ is covered by a local coordinate system $(z, \zeta)$, then one can set $\varphi=\prod\left(z-z\left(P_{i}\right)-\zeta \cdot \zeta\left(P_{i}\right)\right)^{n_{2}}$. Note also that $\log \varphi$ is singlevalued near $C$ owing to $\sum n_{i}=0$.] If now $\mathscr{D}$ is linear equivalent to zero, this means that there exists such a function $\varphi$ defined on the whole of $\mathscr{N}$, so that $\varphi^{-1} D \varphi$ is a field regular in $\mathscr{U}_{2}$. Then by virtue of the relation (18) one has $\int_{C} \alpha\left(h \varphi^{-1} D \varphi\right)=A^{i} B_{i}^{\prime}-B_{i} A^{\prime i}$, where $A^{i}, B_{j}$ are the periods of $\sigma$ and $A^{\prime i}, B_{j}^{\prime}$ are some integers, the periods of $\varphi^{-1} D \varphi$. Consequently, we have proved that the functional (35) equals zero modulo $V$ if $\mathscr{D}$ is linear equivalent to zero.

In order to prove that the map $C l_{0}(\mathscr{N}) \rightarrow J(\mathcal{N})$ constructed above is an isomorphism, we have to construct an inverse map $J(\mathscr{N}) \rightarrow C l_{0}(\mathscr{N})$. Let us take a point in $J(\mathscr{N})$ and let $\bar{\varrho}$ be a $(0,1 / 2)$-field representing it and $L$ be the corresponding line bundle over $\mathscr{N}$. [Thus $\bar{D}_{L}=\bar{D}+\bar{Q}$ and $\bar{\varrho}$ is defined up to equivalence (32) as before.] Let us consider a meromorphic even section $s$ of $L$ which possesses only principal simple zeros and poles making up a divisor $\mathscr{D}$ with $\operatorname{deg} \mathscr{D}=0$. (Remember that $L$ is topologically trivial.) In order to show that this map $J(\mathscr{N}) \rightarrow C l_{0}(\mathscr{N})$ is inverse to the above map $C l_{0}(\mathscr{N}) \rightarrow J(\mathscr{N})$ we must check that the linear functional (35) defined by $\mathscr{D}$ coincides up to the period lattice $V$ with the functional on $\mathscr{A}^{1 / 2}$ defined by the field $\bar{Q}$ [see Eq. (33)]. In a topological trivialization of $L$, when $\bar{D}_{L}=\bar{D}+\bar{\varrho}$, its meromorphic section is represented by a function $s$ satisfying, away from its singular points, the equation $(\bar{D}+\bar{\varrho}) s=0$. Thus, $\bar{\varrho}=-s^{-1} \bar{D} s$ outside the divisor $\mathscr{D}$. Collecting all the notations and facts used above we observe that, in $\mathscr{U}_{1}$, $s$ can be represented in the form $s=\varphi u$ for an invertible function $u$ and meromorphic function $\varphi$ which agrees in $\mathscr{U}_{1}$ with $\mathscr{D}$. Then we have $\bar{\varrho}=-\bar{D} \log u$ in $\mathscr{U}_{1}$. Finally, defining $N_{1}=N \cap \mathscr{U}_{1}, N_{2}=N \cap \mathscr{U}_{2}$ and assuming, additionally that $C$ is contained in $N$, we obtain:

$$
2 \pi i(\bar{\varrho}, \sigma) \equiv 2 i \int_{\mathcal{N}} \bar{\varrho} \sigma d^{2} z d^{2} \zeta=\int_{N_{1}} \beta(\bar{\varrho} \sigma)+\int_{N_{2}} \beta(\bar{\varrho} \sigma) .
$$

Then

$$
\int_{N_{1}} \beta(\bar{\varrho} \sigma)=-\int_{N_{1}} \beta(\bar{D} \log u \cdot \sigma)=\int_{C} \alpha(\log u \cdot \sigma),
$$

and

$$
\int_{N_{2}} \beta(\varrho \bar{\varrho} \sigma)=-\int_{N_{2}} \beta\left(S^{-1} \bar{D}_{S} \cdot \sigma\right)=-\int_{C} \alpha(\log S \cdot \sigma)+\ldots,
$$

where the dots denote $2 \pi i$ times an integer linear combination of periods of $\sigma$. This implies that

$$
\begin{aligned}
2 \pi i(\bar{\varrho}, \sigma) & =\int_{C}[\alpha(\log u \cdot \sigma)-\alpha(\log s \cdot \sigma)]+\ldots \\
& =-\int_{C} \alpha(\log \varphi \cdot \sigma)+\ldots=\int_{C} \alpha\left(h \varphi^{-1} D \varphi\right)+\ldots,
\end{aligned}
$$

for $\sigma=D h$ in $\mathscr{U}_{1}$. Consequently, the functional $\sigma \mapsto(\bar{\varrho}, \sigma)$ coincides with that of Eq. (35) modulo the period lattice. Q.E.D. 
We have thus constructed the required isomorphism $J(\mathscr{N})=C l_{0}(\mathscr{N})$. Note that this proves, in particular, the following useful criterion. A divisor $\mathscr{D}=\sum n_{i} P_{i}$ with $\operatorname{deg} \mathscr{D}=0$ corresponds to a meromorphic function on $\mathscr{N}$ (i.e. $\mathscr{D}$ is linearly equivalent to zero) if and only if

$$
\sum_{i} n_{i} \int_{P_{0}}^{P_{1}} \alpha(\sigma)
$$

equals zero up to periods of $\sigma$, for arbitrary holomorphic type $(1 / 2,0)$ field $\sigma$. This can be, of course, shown also directly, using a technique similar to what was done above.

The manifold of isomorphism classes of holomorphic line bundles is called the Picard manifold. As we have seen, the part of the Picard manifold corresponding to topologically trivial bundles can be identified with the Jacobi manifold $J(\mathscr{N})$ and with the group $C l_{0}(\mathscr{N})$. It is easy to see also that the operation of the tensor product of bundles corresponds then to the addition of divisors. (It is sufficient to note that the divisor of a product of two sections equals the sum of their divisors.)

\section{Computation of Cohomology Groups. Riemann-Roch Theorem}

Let us discuss first the case when all odd parameters of a superconformal manifold $\mathscr{N}$ and of a bundle $L$ over it are zero. That is to say, we suppose that there is a onedimensional complex (ordinary) manifold $N$ with a spin structure and with a holomorphic line bundle $L_{0}$ given on it. Then the superconformal manifold $\mathscr{N}$ and the bundle $L$ can be constructed by means of gluing $(\tilde{z}, \widetilde{\zeta})=\left(u(z), \sqrt{u^{\prime}(z)} \zeta\right)$ and $(\tilde{z}, \tilde{\zeta}, \tilde{l})$ $=\left(u(z), \sqrt{u^{\prime}}(z) \zeta, g(z) l\right)$, where $\tilde{z}=u(z)$ are the transformations which emerge in gluing up the manifold $N$ and $g(z)$ are the transition functions of $L_{0}$. The spin structure on $N$ defines a line bundle by the following rules of gluing:

$$
(z, l) \sim(\tilde{z}, \tilde{l})=\left(u(z),\left[u^{\prime}(z)\right]^{-1 / 2} l\right) .
$$

This bundle will be denoted by $\omega_{0}$. It is easy to see that a holomorphic section $s$ of the bundle $L$ over $\mathscr{N}$ can be written in components as follows:

$$
s(z, \zeta)=s_{0}(z)+\zeta s_{1}(z)
$$

where $s_{0}$ is a section of $L_{0}$ and $s_{1}$ is a section of $L_{0} \otimes \omega_{0}$ on $N$. Consequently, $H^{0}(\mathscr{N}, L)$ is a superspace, with $H^{0}\left(N, L_{0}\right)$ being its even part and $H^{0}\left(N, L_{0} \otimes \omega_{0}\right)$ being its odd part. Similarly, using a component calculation or by means of duality (27), one can verify that the superspace $H^{1}(\mathscr{N}, L)$ is a direct sum of $H^{1}\left(N, L_{0}\right)$ and $\Pi H^{1}\left(N, L_{0} \otimes \omega_{0}\right)$. Let $g$ be the genus of $\mathscr{N}$. If the degree of the bundle $L_{0}$ is negative, $L_{0}$ has no holomorphic sections, i.e. $H^{0}\left(N, L_{0}\right)=0$. On the other hand, if the degree $\geqq g$, then $L_{0}$ does have a non-zero holomorphic section. The degree of $\omega_{0}$ equals $g-1$ and the cases $H^{0}\left(N, \omega_{0}\right)=0$ and $H^{0}\left(N, \omega_{0}\right) \neq 0$ both are possible. In the first case the manifold $N$ with spin structure will be said to be normal. (In algebraic geometry the term "theta characteristic" is used instead of "spin structure." A theta characteristic is called even or odd depending on the parity of the number of linear independent holomorphic sections of $\omega_{0}$. The generic case for an even theta characteristic is the case when this number is zero. It is this case 
which we call the normal case. Usually one says "non-degenerate even theta characteristic".) It follows that if $N$ is a normal manifold, then $H^{0}(\mathscr{N}, L)=0$ in the case when $L_{0}^{*}$ is non-trivial and possesses a non-zero holomorphic section (in particular, when the degree of $L_{0}$ is less than $\left.-g+1\right)$. This statement is valid also when odd parameters are turned on, that is to say, when $\mathscr{N}$ is a normal compact superconformal a manifold (i.e. a supermanifold with underlying manifold being normal) and the bundle $L_{\text {red }}^{*}$ is non-trivial and possesses a non-zero holomorphic section. Roughly speaking, the appearance of odd parameters cannot increase the dimension of a cohomology group. (Under a sufficiently small variation of an ordinary even parameter this dimension also never increases. A variation of odd parameters always can be considered, in a sense, as being infinitesimally small.) Furthermore, in the case considered one can assert that $H^{1}(\mathscr{N}, L)$ is a complex superspace of dimension $(g-d-1 \mid-d)$, where $d$ is the degree of $L_{\text {red }}$ :

$$
\operatorname{dim} H^{1}(\mathcal{N}, L)=(g-d-1 \mid-d) .
$$

For vanishing odd parameters this follows from the ordinary Riemann-Roch theorem,

$$
\operatorname{dim} H^{0}(N, M)-\operatorname{dim} H^{1}(N, M)=\operatorname{deg} M-g+1,
$$

by taking $M=L_{0}$ and $M=L_{0} \otimes \omega_{0}\left(N=\mathscr{N}_{\text {red }}\right)$. In the general case one has to consider the operator $\bar{D}$ acting from $\Gamma(L)$ to $\Gamma(L \otimes \bar{\omega})$. By the above arguments, the kernel of this operator, $\mathscr{A}(L)=H^{0}(\mathscr{N}, L)$, equals zero. Since the dimension of the kernel remains unchanged when varying odd parameters, the same holds for the cokernel, that is for $H^{1}(\mathscr{N}, L)$.

On the other hand, using again Eq. (37) we obtain also the following statement. If $\mathscr{N}$ is a normal compact superconformal manifold, whereas $L_{\text {red }} \otimes \omega_{\text {red }}^{*}$ is nontrivial and possesses a non-zero holomorphic section, then $H^{1}(\mathscr{N}, L)=0$ and $H^{0}(\mathscr{N}, L)$ is a complex superspace of dimension $(d+1-g \mid d)$ :

$$
\operatorname{dim} H^{0}(\mathscr{N}, L)=(d+1-g \mid d) .
$$

Let us point out that according to above arguments Eq. (38) [respectively Eq. (36)] is valid also without assuming that $\mathscr{N}$ is normal, if, however, $H^{1}(\mathscr{N}, L)=0$ [respectively $\left.H^{0}(\mathscr{N}, L)=0\right]$. These results can be applied to the bundle $I=\omega^{k}, \operatorname{deg} \omega^{k}=k(g-1)$. Noticing that $H^{0}\left(\mathscr{N}_{\text {red }}, \omega_{\text {red }}^{n}\right)=0$ for $n<0$, we find that $H^{0}\left(\mathscr{N}, \omega^{k}\right)=0$ for $k \leqq-2$ and that $\operatorname{dim} H^{1}\left(\mathscr{N}, \omega^{k}\right)=((1-k)(g-1) \mid k(1-g))$. Using Eq. (38) we find also that, for $k \geqq 3, \operatorname{dim} H^{0}\left(\mathscr{N}, \omega^{k}\right)=((k-1)(g-1) \mid k(g-1))$, whereas $H^{1}\left(\mathscr{N}, \omega^{k}\right)=0$. In the case of a normal superconformal manifold $\mathscr{N}$ one can compute also cohomology groups $H^{p}\left(\mathscr{N}, \omega^{k}\right)$ for $k=0,1$. (That is to say, when either $L$ is a trivial bundle $\mathcal{O}=\omega^{0}$ or it is $\omega$.) In this case one has $\operatorname{dim} H^{0}(\mathscr{N}, \mathcal{O})$ $=(1 \mid 0)$ and $\operatorname{dim} H^{0}(\mathscr{N}, \omega)=(0 \mid g)$. Indeed, for vanishing odd parameters, holomorphic functions on $\mathscr{N}$ (i.e. sections of the trivial bundle $\mathcal{O}$ ) are related to holomorphic functions on $\mathscr{N}_{\text {red }}$ and holomorphic sections of $\omega_{\text {red }}$. The former ones are constants, for $\mathscr{N}_{\text {red }}$ is compact, while the latter ones are necessarily zero, for $\mathscr{N}$ is normal. The constants continue to be holomorphic sections of $\mathscr{O}$ in the presence of non-vanishing odd parameters as well. This means that the dimension $(1 \mid 0)$ of $H^{0}(\mathcal{N}, \mathcal{O})$ doesn't decrease and, hence, remains the same after turning on odd parameters. Since the dimension of the kernel, $H^{0}(\mathscr{N}, \mathcal{O})$, of $\bar{D}$ acting from $\Gamma(\mathcal{O})$ to 
$\Gamma(\bar{\omega})$ is thus independent of odd parameters, so are $\operatorname{dim} H^{1}(\mathscr{N}, \mathcal{O})$ and, hence, $\operatorname{dim} H^{0}(\mathcal{N}, \omega)$ by virtue of the duality (27). It remains to observe that for vanishing odd parameters the group $H^{0}(\mathcal{N}, \omega)$ reduces to a trivial group, $H^{0}\left(\mathcal{N}_{\text {red }}, \omega_{\text {red }}\right)$, (by virtue of $\mathscr{N}$ being normal $)$ and to the group $H^{0}\left(\mathcal{N}_{\text {red }}, \omega_{\text {red }}^{2}\right)$ of dimension $g$. [Note that holomorphic sections of $\omega_{\text {red }}^{2}$ are nothing but holomorphic $(1,0)$-forms, that is abelian differentials.] All this implies, in particular, that a holomorphic type $(1 / 2,0)$ field on a normal manifold can be specified completely by its periods with respect to the cycles $a^{1}, \ldots, a^{g}$ (i.e. by its $A$-periods). Thus, if all the $A$-periods of a holomorphic type $(1 / 2,0)$ field on a normal manifold vanish, then the field itself equals zero.

The results proved above can also be derived from a general theorem, the super analog of the Riemann-Roch theorem. This gives the following. If $H^{0}(\mathcal{N}, L)$ and $H^{1}(\mathscr{N}, L)$ can be considered as superspaces, then

$$
\operatorname{dim} H^{0}(\mathscr{N}, L)-\operatorname{dim} H^{1}(\mathscr{N}, L)=(d+1-g \mid d),
$$

where $d$ is the degree of $L, g$ is the genus of the superconformal manifold $\mathscr{N}$. In the absence of odd parameters this follows from the ordinary Riemann-Roch theorem. In the general case, one has to use the fact that the index of the operator $\bar{D}$ remains unchanged under variation of parameters. (Remember that the index of an operator is defined as the difference of dimensions of its kernel and cokernel.)

\section{Appendix}

Let $\Lambda$ be a real Grassmann algebra. An even $\Lambda$-point of the $(p \mid q)$-dimensional superspace $\mathbb{R}^{p \mid q}$ means a row of $p$ even and $q$ odd elements of $\Lambda$. An odd $\Lambda$-point is given by a row of $p$ odd and $q$ even elements of $\Lambda$. Taking $\Lambda$ to be a complex Grassmann algebra we obtain similarly the definition of a $\Lambda$-point of the complex $(p \mid q)$-dimensional superspace $\mathbb{C}^{p \mid q}$. The set of even $\Lambda$-points of $\mathbb{R}^{p \mid q}$ or, respectively, of $\mathbb{C}^{p \mid q}$ will be denoted by $\mathbb{R}_{A}^{p \mid q}, \mathbb{C}_{A}^{p \mid q}$. For a superspace $E$ we denote a superspace with inverse parity by $\Pi E$. $\Pi E$ possesses the same set of $\Lambda$-points except for their parities which are renamed. Those points which were even in $E$ are called odd in $\Pi E$ and vice versa. Thus, for instance, $\Pi \mathbb{R}^{p \mid q}=\mathbb{R}^{q \mid p}$. If $\mathscr{U}$ is a domain in $\mathbb{R}^{p}$, then the subset of $\mathbb{R}_{\Lambda}^{p \mid q}$ consisting of rows $\left(x^{1}, \ldots, x^{p}, \xi^{1}, \ldots, \xi^{q}\right)$ with $\left(m\left(x^{1}\right), \ldots, m\left(x^{p}\right)\right) \in \mathscr{U}$ will be called a $\Lambda$-superdomain $\mathscr{U}_{A}^{q}$. [We have used here the notation $m(x)$ for the numerical part of $x \in \Lambda$.] For a complex domain $\mathscr{U} \subset \mathbb{C}^{p}$, the corresponding $\Lambda$-superdomain $\mathscr{U}_{\Lambda}^{q} \subset \mathbb{C}_{\Lambda}^{p \mid q}$ is defined analogously. A $\Lambda$-map of $\mathbb{R}_{\Lambda}^{p \mid q}$ into $\mathbb{R}_{\Lambda}^{r \mid s}$ means a map of the following form:

$$
\begin{aligned}
y^{n} & =\sum f_{\alpha_{1} \ldots \alpha_{k}}^{n}\left(x^{1}, \ldots, x^{p}\right) \xi^{\alpha_{1}} \ldots \xi^{\alpha_{k}}, \\
\eta^{\mu} & =\sum g_{\alpha_{1} \ldots \alpha_{k}}^{\mu}\left(x^{1}, \ldots, x^{p}\right) \xi^{\alpha_{1}} \ldots \xi^{\alpha_{k}},
\end{aligned}
$$

where $\left(y^{1}, \ldots, y^{r}, \eta^{1}, \ldots, \eta^{s}\right) \in \mathbb{R}_{\Lambda}^{r \mid s},\left(x^{1}, \ldots, x^{p}, \xi^{1}, \ldots, \xi^{q}\right) \in \mathbb{R}_{\Lambda}^{p \mid q}, f_{\alpha_{1} \ldots \alpha_{k}}^{n}$ is a $\Lambda$-function of parity equal to the parity of the number $k$, and $g_{\alpha_{1} \ldots \alpha_{k}}^{\mu}$ is a $A$-function of parity opposite to that of $k$. (A $A$-function is by definition a linear combination of ordinary smooth functions with coefficients in $A$ and with even elements $x^{1}, \ldots, x^{p}$ of $\Lambda$ substituted for their arguments.) One can define analogously $\Lambda$-maps of complex superspaces and 1 -maps of superdomains. A 1 -manifold can be 
understood as a manifold glued up from $\Lambda$-superdomains by means of $\Lambda$-maps. In the text of this paper supermanifolds can always be understood as $\Lambda$-manifolds, their points can be understood as even $\Lambda$-points, and maps can be understood as $\Lambda$-maps for a fixed Grassmann algebra $\Lambda$. Another approach to the definition of a supermanifold implies consideration of a family of $\Lambda$-manifolds for all $\Lambda$ 's, every two of them being related in a certain way (cf. [16]). As a matter of course, all the main results remain valid for the latter definition as well.

A $\Lambda$-manifold can be singled out in $\mathbb{R}_{A}^{p \mid q}$ (or $\mathbb{C}_{A}^{p \mid q}$ ) by a system of equations, say,

$$
f(z)=0,
$$

where $f$ is a $\Lambda$-map of $\mathbb{R}^{p \mid q}$ into $\mathbb{R}^{p-r \mid q-s}$. Equation (A2) defines, in general, an $(r \mid s)$-dimensional $\Lambda$-manifold. It is worth pointing out, however, that this is not always the case. For example, a system of linear equations,

$$
\begin{aligned}
& a_{i}^{n} x^{i}+\alpha_{\gamma}^{n} \xi^{\gamma}=0, \\
& \beta_{i}^{\mu} x^{i}+b_{\gamma}^{\mu} \xi^{\gamma}=0,
\end{aligned}
$$

with $i=1, \ldots, p ; \gamma=1, \ldots, q ; n=1, \ldots, p-r ; \mu=1, \ldots, q-s$, defines an $(r \mid s)$ dimensional $\Lambda$-manifold only if the numerical parts of the matrices $\left(a_{i}^{n}\right),\left(b_{\gamma}^{\mu}\right)$ are of maximal rank [i.e. $\left.\operatorname{rank} m\left(a_{i}^{n}\right)=p-r, \operatorname{rank} m\left(b_{\gamma}^{\mu}\right)=q-s\right]$. In the case of a non-linear system of equations similar constraints must be imposed on principal linear parts.

From every $\Lambda$-map $f$ of superdomains one can make a map $f_{\text {red }}$ of ordinary domains by reducing all the nilpotents, that is replacing all the elements of Grassmann algebra by their numerical parts. To every $\Lambda$-manifold $\mathscr{X}$ one can relate an ordinary manifold $\mathscr{X}_{\text {red }}$ called the underlying manifold of $\mathscr{X}$. The manifold $\mathscr{X}_{\text {red }}$ can be obtained also by reducing the nilpotents. More precisely, if $\mathscr{X}$ is glued up from $\Lambda$-superdomains by means of some $\Lambda$-maps, then $\mathscr{X}_{\text {red }}$ must be glued up from ordinary domains by means of corresponding reduced maps. Moreover, for every $\Lambda$-map $f$ of $\Lambda$-manifold $\mathscr{X}$ into $\Lambda$-manifold $\mathscr{Y}$ there is a corresponding map $f_{\text {red }}: \mathscr{X}_{\text {red }} \rightarrow \mathscr{Y}_{\text {red }}$. One has also a natural map $\mathscr{X} \rightarrow \mathscr{X}_{\text {red }}$. A point of $\mathscr{X}_{\text {red }}$, which results under this map from a $A$-point $P$ of $\mathscr{X}$, will be denoted by $P_{\text {red. }}{ }^{3}$

In this paper we deal, in particular, with cohomology groups $H^{0}(\mathscr{N}, L)$ and $H^{1}(\mathscr{N}, L)$ with coefficients in a bundle $L$ over a superconformal manifold $\mathscr{N}$. These groups we regard as $\Lambda$-manifolds (or superspaces, according to the terminology used mainly in the text). Such a treatment, however, may sometimes be impossible, for the equation $\bar{D} \varphi=0$, which distinguish elements of $H^{0}(\mathscr{N}, L)$ (i.e. holomorphic sections of $L$ ) from an infinite-dimensional superspace of all sections, may not satisfy the conditions described above. In the latter case we say that $H^{0}(\mathscr{N}, L)$ cannot be considered as a superspace. An analogous situation may also take place for $H^{1}(\mathscr{N}, L)$. In other words, the groups $H^{p}(\mathscr{N}, L)$ always are $\Lambda$-modules, but these $\Lambda$-modules may not be free in some cases. If not pointed out otherwise, all the cohomology groups considered are understood to be superspaces.

In the text of this paper we often say "manifold" instead of "supermanifold" (or, more accurately, instead of " $\Lambda$-manifold"), "space" instead of "superspace," and so

\footnotetext{
${ }^{3}$ Note that this set-theoretical map, $\mathscr{X} \rightarrow \mathscr{X}_{\mathrm{red}}$, cannot be considered as a $\Lambda$-map of supermanifolds, since it maps $A$-points of $\mathscr{X}$ into $\mathbb{R}$-points of $\mathscr{X}_{\text {red }}$. This means, in particular, that the map $\mathscr{X} \rightarrow \mathscr{X}_{\text {red }}$ does not induce a map of functions on $\mathscr{X}_{\text {red }}$ into $A$-functions on $\mathscr{X}$
} 
on. The term "determinant" always means the superdeterminant (i.e. the Berezinian).

In Sect. 4 we have to put certain constraints on the meromorphic functions to be considered. This restriction could be avoided if one used a more general definition of divisors. On an arbitrary $(m \mid n)$-dimensional complex algebraic supermanifold $\mathscr{N}$ a divisor is defined (according to Cartier) by the following set of data. Let $\left\{\mathscr{U}_{i}\right\}$ be a covering of $\mathscr{N}$ by open superdomains, $\varphi_{i}$ be even meromorphic functions on $\mathscr{U}_{i}$, such that $\left(\varphi_{i}\right)_{\text {red }} \neq 0$ and that $g_{i j}=\varphi_{i} / \varphi_{j}$ are holomorphic functions on $\mathscr{U}_{i} \cap \mathscr{U}_{j}$ with nowhere vanishing $\left(g_{i j}\right)_{\text {red }}$. The corresponding divisor is defined then as a class of sets $\left\{\varphi_{i}\right\}$ with respect to equivalence $\varphi_{i} \sim g_{i} \varphi_{i}$ if $g_{i}$ are holomorphic functions in $\mathscr{U}_{i}$ with $\left(g_{i}\right)_{\text {red }} \neq 0$ in $\left(\mathscr{U}_{i}\right)_{\text {red. }}$. Such a divisor represents, roughly speaking, a linear combination of $(m-1 \mid n)$-dimensional submanifolds with integer coefficients. Note also that every such divisor is related to a line bundle defined by transition functions $g_{i j}$, which appeared above, and that the classes of divisors are in one-to-one correspondence with isomorphism classes of line bundles. If $\mathscr{N}$ is a (1|1)-dimensional superconformal manifold, then Cartier divisors may correspond to (0|1)-dimensional complex submanifolds. Had we used in this case Cartier divisors, we get, in fact, the same results as in Sect. 4. This is indicated, in particular, by the following correspondence. Every divisor defined as in Sect. 4 (i.e. a linear combination of points) corresponds to a certain Cartier divisor. If, for instance, the former is a single point $P=\left(z_{0}, \zeta_{0}\right)$, then the latter is defined by the function $\left(z-z_{0}-\zeta \zeta_{0}\right)$ in a domain containing $P$ and by functions equal to one in other domains. In other words, this Cartier divisor corresponds to a (0|1)-dimensional submanifold $\left\{z-z_{0}-\zeta \zeta_{0}=0\right\}$. Conversely, if some Cartier divisor is given locally, for example, by an even holomorphic function $\varphi$, such that $\varphi_{\text {red }}$ has a single simple zero [or by a (0|1)-dimensional submanifold $\{\varphi=0\}$ ], then the corresponding divisor in the sense of Sect. 4 contains the point defined by equations $\varphi=0, D \varphi=0$, i.e. the principal zero of $\varphi$, with the unit coefficient. (Note that although we have no complete correspondence between the divisors of two kinds, this is inessential, for the groups of classes of linear equivalent divisors are isomorphic. Indeed these groups are both isomorphic to the group of classes of line bundles.)

The above connections between divisors in the sense of Cartier and divisors in the sense of Sect. 4 is analogous to the correspondence between $(1,0)$-forms and fields of type $(1 / 2,0)$ described in Sect. 1 . Thus, for example, one could define the periods as the integrals of type $(1 / 2,0)$ fields over $(1 \mid 1)$-dimensional real cycles. In this case, to derive bilinear relations (8), (9) in Sect. 2 one had to use the Stokes' theorem for integral forms instead of differential forms.

\section{References}

1. Polyakov, A.M.: Quantum geometry of bosonic strings. Phys. Lett. 103 B, 207 (1981)

2. Baranov, M.A., Schwarz, A.S.: Multiloop contribution to string theory. Pis'ma ZhETP 42:8, 340 (1985) [JETP Lett. 42, 419 (1986)]

3. Friedan, D.: Notes on string theory and two dimensional conformal field theory. In: Workshop on Unified String Theories (ITP Santa Barbara 1985). Green, M., Gross, D. (eds.), p. 162. Singapore: World Scientific 1986 
4. Baranov, M.A., Frolov, I.V., Schwarz, A.S.: Geometry of two dimensional superconformal field theories. Teor. Mat. Fiz. 70, 92 (1987)

5. Moore, G., Nelson, P., Polchinski, J.: Strings and supermoduli. Phys. Lett. 169 B, 47 (1986)

6. Belavin, A.A., Knizhnik, V.G.: Algebraic geometry and the geometry of quantum strings. Phys. Lett. 168 B, 201 (1986)

7. Beilinson, A.A., Manin, Yu.I.: The Mumford form and the Polyakov measure in string theory. Commun. Math. Phys. 107, 359 (1986)

8. Baranov, M.A., Manin, Yu.I., Frolov, I.V., Schwarz, A.S.: Multiloop contribution in fermion string. Yad. Fiz. 43, 1053 (1986)

9. Baranov, M.A., Manin, Yu.I., Frolov, I.V., Schwarz, A.S.: A super analog of the Selberg trace formula and multiloop contribution for fermion string. Commun. Math. Phys. 109, 350 (1987)

10. Voronov, A.A.: A formula for Mumford measure in superstring theory. Funk. Anal. i Prilozen. 21, $312(1987)$

11. Griffiths, P., Harris, J.: Principles of algebraic geometry. New York: Wiley (1978) Alvarez-Gaumé, L., Moore, G., Vafa, C.: Theta functions, modular invariance, and strings. Commun. Math. Phys. 106, 1 (1986)

12. Baranov, M.A., Schwarz, A.S.: On the multiloop contribution to the string theory. Int. J. Mod. Phys. A 3, 28 (1987)

13. Rosly, A.A., Schwarz, A.S., Voronov, A.A.: Superconformal geometry and string theory. Preprint ITEP (1987)

14. Schwarz, A.S.: The partition function of a degenerate functional. Commun. Math. Phys. 67, 1 (1979)

15. Schwarz, A.S., Tyupkin, Yu.S.: Quantization of antisymmetric tensors and Ray-Singer torsion. Nucl. Phys. B 242, 436 (1984)

16. Schwarz, A.S.: To the definition of superspace. Teor. Mat. Fiz. 60, 37 (1984)

Communicated by A. Jaffe

Received December 23, 1987; in revised form May 5, 1987 